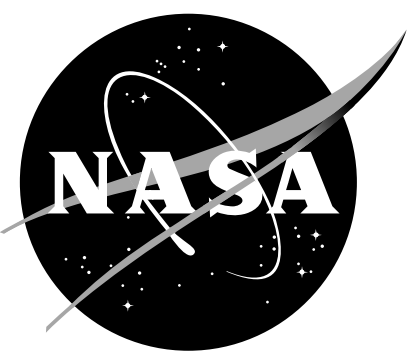

\title{
A Systematic Methodology for Constructing High-Order Energy-Stable WENO Schemes
}

Nail K. Yamaleev

Department of Mathematics, North Carolina A\&ST State University

Greensboro, North Carolina

Mark H. Carpenter

Langley Research Center, Hampton, Virginia 
Since its founding, NASA has been dedicated to the advancement of aeronautics and space science. The NASA Scientific and Technical Information (STI) Program Office plays a key part in helping NASA maintain this important role.

The NASA STI Program Office is operated by Langley Research Center, the lead center for NASA's scientific and technical information. The NASA STI Program Office provides access to the NASA STI Database, the largest collection of aeronautical and space science STI in the world. The Program Office is also NASA's institutional mechanism for disseminating the results of its research and development activities. These results are published by NASA in the NASA STI Report Series, which includes the following report types:

- TECHNICAL PUBLICATION. Reports of completed research or a major significant phase of research that present the results of NASA programs and include extensive data or theoretical analysis. Includes compilations of significant scientific and technical data and information deemed to be of continuing reference value. NASA counterpart of peer-reviewed formal professional papers, but having less stringent limitations on manuscript length and extent of graphic presentations.

- TECHNICAL MEMORANDUM. Scientific and technical findings that are preliminary or of specialized interest, e.g., quick release reports, working papers, and bibliographies that contain minimal annotation. Does not contain extensive analysis.

- CONTRACTOR REPORT. Scientific and technical findings by NASA-sponsored contractors and grantees.
- CONFERENCE PUBLICATION. Collected papers from scientific and technical conferences, symposia, seminars, or other meetings sponsored or co-sponsored by NASA.

- SPECIAL PUBLICATION. Scientific, technical, or historical information from NASA programs, projects, and missions, often concerned with subjects having substantial public interest.

- TECHNICAL TRANSLATION. Englishlanguage translations of foreign scientific and technical material pertinent to NASA's mission.

Specialized services that complement the STI Program Office's diverse offerings include creating custom thesauri, building customized databases, organizing and publishing research results ... even providing videos.

For more information about the NASA STI Program Office, see the following:

- Access the NASA STI Program Home Page at http://www.sti.nasa.gov

- E-mail your question via the Internet to help@sti.nasa.gov

- Fax your question to the NASA STI Help Desk at (301) 621-0134

- Phone the NASA STI Help Desk at (301) 621-0390

- Write to: NASA STI Help Desk NASA Center for AeroSpace Information 7115 Standard Drive Hanover, MD 21076-1320 


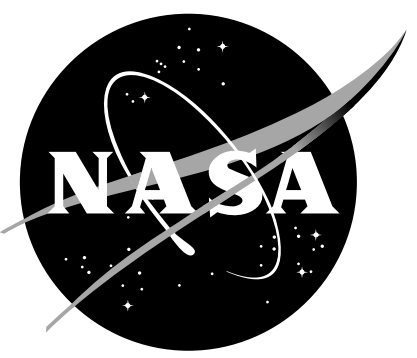

\section{A Systematic Methodology for Constructing High-Order Energy-Stable WENO Schemes}

Nail K. Yamaleev

Department of Mathematics, North Carolina A\&ST State University

Greensboro, North Carolina

Mark H. Carpenter

Langley Research Center, Hampton, Virginia

National Aeronautics and

Space Administration

Langley Research Center

Hampton, Virginia 23681-2199

October 2008 


\section{Acknowledgments}

This work was partially performed while the second author was in residence at Technical University of Delft, Delft, The Netherlands (technical monitor: Professor Hester Bijl).

Available from:

NASA Center for AeroSpace Information (CASI)

7115 Standard Drive

Hanover, MD 21076-1320

(301) 621-0390
National Technical Information Service (NTIS) 5285 Port Royal Road

Springfield, VA 22161-2171

(703) 605-6000 


\section{Abstract}

A third-order Energy Stable Weighted Essentially Non-Oscillatory (ESWENO) finite difference scheme developed by Yamaleev and Carpenter (AIAA 2008-2876, 2008) was proven to be stable in the energy norm for both continuous and discontinuous solutions of systems of linear hyperbolic equations. Herein, a systematic approach is presented that enables "energy stable" modifications for existing WENO schemes of any order. The technique is demonstrated by developing a one-parameter family of fifth-order upwindbiased ESWENO schemes; ESWENO schemes up to eighth order are presented in the appendix. New weight functions are also developed that provide (1) formal consistency, (2) much faster convergence for smooth solutions with an arbitrary number of vanishing derivatives, and (3) improved resolution near strong discontinuities.

\section{Contents}

1 Introduction $\quad 1$

2 Energy Estimates $\quad 2$

3 Fifth- and Sixth-order WENO Schemes 4

4 A General Approach to Constructing High-Order Energy-Stable Schemes $\quad 7$

5 Consistency Analysis 9

5.1 Necessary and Sufficient Conditions for Consistency of WENO Schemes . . . . . . . . . 9

5.2 Sufficient Conditions for Consistency of ESWENO Schemes . . . . . . . . . . . . . . 10

5.3 Consistency of the ESWENO scheme with New Weights . . . . . . . . . . . . . 12

6 Numerical Results $\quad \mathbf{1 6}$

6.1 Scalar Linear Wave Equation $\ldots \ldots \ldots \ldots \ldots \ldots \ldots \ldots$

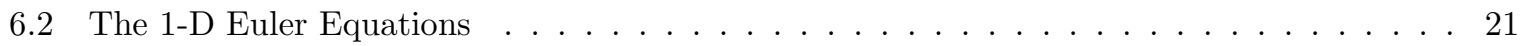

7 Conclusions $\quad 24$

8 Appendix $\quad \mathbf{2 6}$

8.1 Existence of Symmetric Decomposition . . . . . . . . . . . . . . . . . . 26

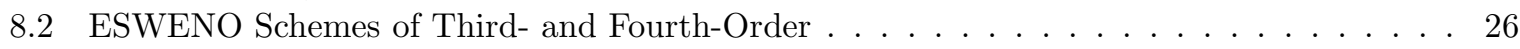

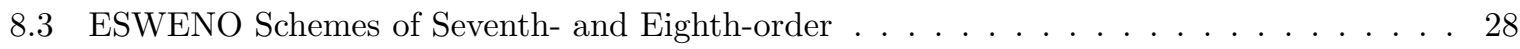

\section{Introduction}

A new, third-order weighted essentially nonoscillatory scheme (called Energy-Stable WENO) has recently been proposed and developed by the authors of the present paper [1]. In reference [1], we prove that the third-order ESWENO scheme is energy stable, that is, stable in an $L_{2}$ energy norm, for systems of linear hyperbolic equations with both continuous and discontinuous solutions. Stability is explicitly achieved (by construction) by requiring that the ESWENO scheme satisfies a nonlinear summation-byparts (SBP) condition at each instant in time. Thus, $L_{2}$ strict stability is attained without the need for a total variation bounded (TVB) flux reconstruction or a large-time-step constraint [2], [3] and [4]. Herein, we generalize and extend the third-order ESWENO methodology [1] to an arbitrary order of accuracy. Similar to the third-order ESWENO scheme, the new families of higher order (up to eighth order) ESWENO schemes are provably stable in the energy norm and retain the underlying WENO characteristics of the background schemes. Numerical experiments demonstrate that the new family 
of ESWENO schemes provides the design order of accuracy for smooth problems and delivers stable essentially nonoscillatory solutions for problems with strong discontinuities.

Another issue that is addressed in this paper is the consistency of the new class of ESWENO schemes. The consistency of any WENO-type scheme fully depends on a proper choice of the weight functions. On one hand, for smooth solutions the weights should provide a rapid convergence of the WENO scheme to the corresponding underlying linear scheme. On the other hand, the weights should effectively bias the stencil away from strong discontinuities. The high-order upwind-biased WENO schemes with conventional smoothness indicators that are presented in reference [2] are too dissipative for solving problems with a large amount of structure in the smooth part of the solution, such as direct numerical simulations of turbulence, or aeroacoustics[5], [6]. Furthermore, as has been shown in references [7] and [8], the classical weight functions of the fifth-order WENO scheme fail to provide the design order of convergence near smooth extrema, where the first derivative of the solution becomes equal to zero. New approaches are proposed in references [7] and [8] to improve the error convergence near the critical points. Although these new weight functions recover the fifth order of convergence of the WENO scheme near smooth extrema, the problem persists if the first- and second-order derivatives vanish simultaneously [8]. An attempt to resolve this loss of accuracy is presented in reference [8]. This proposed resolution provides only a partial remedy for the problem; the same degeneration in the order of convergence occurs if at least the first three derivatives become equal to zero. To fully resolve this problem, we propose new weights to provide faster error convergence than those presented in reference [8], and impose some constraints on the weight parameters to guarantee that the WENO and ESWENO schemes are design-order accurate for sufficiently smooth solutions with an arbitrary number of vanishing derivatives.

This paper is organized as follows. In section 2, energy estimates for the continuous and corresponding discrete wave equations are presented. In section 3, we present a one-parameter family of fifth-order WENO schemes; one value of the parameter yields a central scheme that converges with sixth-order accuracy. In section 4, we present a systematic methodology for constructing ESWENO schemes of any order and demonstrate the methodology by transforming the family of WENO schemes presented in section 3, into a family of fifth-order ESWENO schemes. In section 5, we analyze the consistency of the new class of ESWENO schemes and we derive sufficient conditions for the weights functions that ensure that the ESWENO schemes are design-order accurate regardless of the number of vanishing derivatives in the solution. The tuning parameters in the weight functions are also optimized. In section 6 , we present numerical experiments that corroborate our theoretical results. We summarize and draw conclusions in section 7 .

\section{$2 \quad$ Energy Estimates}

Consider a linear, scalar wave equation

$$
\begin{aligned}
& \frac{\partial u}{\partial t}+\frac{\partial f}{\partial x}=0, \quad f=a u, \quad t \geq 0, \quad 0 \leq x \leq 1 \\
& u(0, x)=u_{0}(x)
\end{aligned}
$$

where $a$ is a constant and $u_{0}(x)$ is a bounded piecewise continuous function. Without loss of generality, assume that $a \geq 0$, and further assume that the problem is periodic on the interval $0 \leq x \leq 1$. Applying the energy method to equation (1) leads to

$$
\frac{d}{d t}\|u\|_{L_{2}}^{2}=0
$$

where $\|\cdot\|_{L_{2}}$ is the continuous $L_{2}$ norm. Thus, the continuous problem defined in equation (1) is neutrally stable.

We now develop using mimetic techniques (see [1] or [9]) a class of discrete spatial operators that is neutrally stable or dissipative. The continuous target operator used for this development is the following 
singular perturbed wave equation:

$$
\begin{aligned}
& \frac{\partial u}{\partial t}+\frac{\partial f}{\partial x}=\sum_{n=1}^{N}(-1)^{n-1} \frac{\partial^{n}}{\partial x^{n}}\left(\mu \frac{\partial^{n} u}{\partial x^{n}}\right), \quad f=a u, \quad t \geq 0, \quad 0 \leq x \leq 1 \\
& u(0, x)=u_{0}(x)
\end{aligned}
$$

where $\mu=\mu(u)$ is a non-negative $C^{\infty}$ function of $u$. As before, we assume that equation (3) is subject to periodic boundary conditions. Our goal is to match each spatial term in equation (3) with an equivalent discrete term that maintains neutral stability (or dissipates) of the discrete energy norm.

We begin by showing that the terms on the right-hand side of equation (3) are dissipative thereby ensuring stability. Multiplying equation (3) by $u$ and integrating it over the entire domain yields

$$
\frac{1}{2} \frac{d}{d t}\|u\|_{L_{2}}^{2}+\left.\frac{1}{2} a u^{2}\right|_{0} ^{1}=\sum_{n=1}^{N} \int_{0}^{1}(-1)^{n-1} u \frac{\partial^{n}}{\partial x^{n}}\left(\mu \frac{\partial^{n} u}{\partial x^{n}}\right) d x .
$$

Integrating each term on the right-hand side by parts and accounting for periodic boundary conditions yields the following energy estimate:

$$
\frac{d}{d t}\|u\|_{L_{2}}^{2}=-2 \sum_{n=1}^{N} \int_{0}^{1} \mu(u)\left(\frac{\partial^{n} u}{\partial x^{n}}\right)^{2} d x \leq 0 .
$$

All the perturbation terms included in equation (3) provide dissipation of energy.

Turning now to the discrete case, we define a uniform grid $x_{j}=j \Delta x, j=\overline{0, J}$, with $\Delta x=1 / J$. On this grid, we define a flux $\overline{\mathbf{f}}=a \overline{\mathbf{u}}$ and its derivative $\overline{\mathbf{f}}_{x}=a \overline{\mathbf{u}}_{x}$, where $\overline{\mathbf{u}}=\left[u\left(x_{0}, t\right), \ldots, u\left(x_{J}, t\right)\right]^{T}$ and $\overline{\mathbf{u}}_{x}=\left[u_{x}\left(x_{0}, t\right), \ldots, u_{x}\left(x_{J}, t\right)\right]^{T}$ are projections of the continuous solution and its derivative onto the computational grid. Next, we define a $p$ th-order approximation for the first-order derivative term in equation (1) as

$$
\frac{\partial \overline{\mathbf{f}}}{\partial x}=D \overline{\mathbf{f}}+O\left(\Delta x^{p}\right)
$$

Placing a mild restriction on the generality of the derivative operator (see [1] or [9]), the matrix $D$ can be expressed in the following form:

$$
\begin{array}{rll}
D=P^{-1}[Q+R] & ; \quad & Q+Q^{T}=0 \\
R=R^{T} & ; \quad \mathbf{v}^{T} R \mathbf{v} \geq 0 \\
P=P^{T} & ; \quad \mathbf{v}^{T} P \mathbf{v}>0
\end{array}
$$

for any real vector $\mathbf{v} \neq \mathbf{0}$. By choosing the matrix $R$ as a discrete analog of the dissipation operator in equation (3), we have

$$
R=\sum_{n=1}^{N} D_{1}^{n} \Lambda\left[D_{1}^{n}\right]^{T}
$$

where $\Lambda$ is a diagonal positive semidefinite matrix and $D_{1}$ is the difference matrix

$$
D_{1}=\left[\begin{array}{cccc} 
& \ddots & & 0 \\
& -1 & 1 & \\
0 & & & \ddots
\end{array}\right] \text {. }
$$

By using the SBP operators [eqs. (6)-(8)], the semi-discrete counterpart of equation (1) becomes

$$
\frac{\partial \mathbf{u}}{\partial t}+P^{-1} Q \mathbf{f}=-\sum_{n=1}^{N} P^{-1} D_{1}^{n} \Lambda\left[D_{1}^{n}\right]^{T} \mathbf{f}
$$


where $\mathbf{f}=a \mathbf{u}, \mathbf{u}=\left[u_{0}(t), u_{1}(t), \ldots, u_{J}(t),\right]^{T}$ is the discrete approximation of the solution $u$ of equation (1), and $Q$ and $\Lambda$ are nonlinear matrices (i.e., $Q=Q(\mathbf{u})$ and $\Lambda=\Lambda(\mathbf{u})$. To show that the above finite-difference scheme is stable, the energy method is used. Multiplying equation (9) with $\mathbf{u}^{T} P$ yields

$$
\frac{1}{2} \frac{d}{d t}\|\mathbf{u}\|_{P}^{2}+a \mathbf{u}^{T} Q \mathbf{u}=-a \sum_{n=1}^{N}\left(\left[D_{1}{ }^{n}\right]^{T} \mathbf{u}\right)^{T} \Lambda\left[D_{1}{ }^{n}\right]^{T} \mathbf{u}
$$

where $\|\cdot\|_{P}$ is the $P$ norm (i.e., $\|\mathbf{u}\|_{P}^{2}=\mathbf{u}^{T} P \mathbf{u}$ ). Adding equation (10) to its transpose yields

$$
\frac{d}{d t}\|\mathbf{u}\|_{P}^{2}+a \mathbf{u}^{T}\left(Q+Q^{T}\right) \mathbf{u}=-2 a \sum_{n=1}^{N}\left(\left[D_{1}{ }^{n}\right]^{T} \mathbf{u}\right)^{T} \Lambda\left[D_{1}{ }^{n}\right]^{T} \mathbf{u} .
$$

If we account for periodic boundary conditions and the skew-symmetry of $Q$, then the second term on the left-hand side vanishes, and the energy estimate becomes

$$
\frac{d}{d t}\|\mathbf{u}\|_{P}^{2}=-2 a \sum_{n=1}^{N}\left(\left[D_{1}^{n}\right]^{T} \mathbf{u}\right)^{T} \Lambda\left[D_{1}{ }^{n}\right]^{T} \mathbf{u} \leq 0
$$

The right-hand side of equation (12) is nonpositive because the diagonal matrix $\Lambda$ is positive semidefinite $\left[\mathbf{v}^{T} \Lambda \mathbf{v} \geq 0\right.$ for all real $\mathbf{v}$ of length $\left.(J+1)\right]$ and $a \geq 0$; thus the stability of the finite-difference scheme given by equation (9) is assured. This result can be summarized in the following theorem:

Theorem 1. The approximation [eq. (9)] of the problem [eq. (1)] is stable if equations [(6)-(8)] hold.

Remark 1. Despite the fact that the initial boundary value problem [eq. (1)] is linear, the finite-difference scheme [eq. (9)] constructed for approximation of equation (1) is nonlinear, because the matrices $Q$ and $\Lambda$ (and in principle $P$ ) are assumed to depend on the discrete solution $\mathbf{u}$.

Remark 2. The only constraints that are imposed on the matrix $Q$ and the diagonal matrix $\Lambda$ are the skew-symmetry of the former and positive semidefiniteness of the later. No other assumptions have been made about a specific form of the matrices $Q$ and $\Lambda$ to guarantee the stability of the finite-difference scheme [eq. (9)].

Remark 3. The discrete operators that are defined by equations [(6)-(8)] are similar in form to those that are used for conventional SBP operators (See refs. $[9,10]$ ). What is new, however, is the fact that the matrices $Q$ and $\Lambda$ depend on $\mathbf{u}$.

Next, a new one-parameter family of fifth-order WENO schemes is developed, and then is used as the starting point in the development of a family of "energy-stable" WENO schemes. Great care is exercised in the developing the WENO schemes to ensure that design-order accuracy is achieved in the vicinity of smooth extrema.

\section{Fifth- and Sixth-order WENO Schemes}

Any conventional high-order WENO finite-difference scheme for the scalar one-dimensional wave equation (1) can be written in the following semidiscrete form:

$$
\frac{d u_{j}}{d t}+\frac{\hat{f}_{j+\frac{1}{2}}-\hat{f}_{j-\frac{1}{2}}}{\Delta x}=0
$$

For the fifth-order WENO scheme that is presented in reference [2], the numerical flux $\hat{f}_{j+\frac{1}{2}}$ is computed as a convex combination of three third-order fluxes defined on the following three-point stencils: $S_{L L}=$ $\left\{x_{j-2}, x_{j-1}, x_{j}\right\}, S_{L}=\left\{x_{j-1}, x_{j}, x_{j+1}\right\}$, and $S_{R}=\left\{x_{j}, x_{j+1}, x_{j+2}\right\}$. (See figure 1.) Note that this set of stencils is not symmetric with respect to the $\left(j+\frac{1}{2}\right)$ point; thus, the fifth-order WENO scheme is biased 


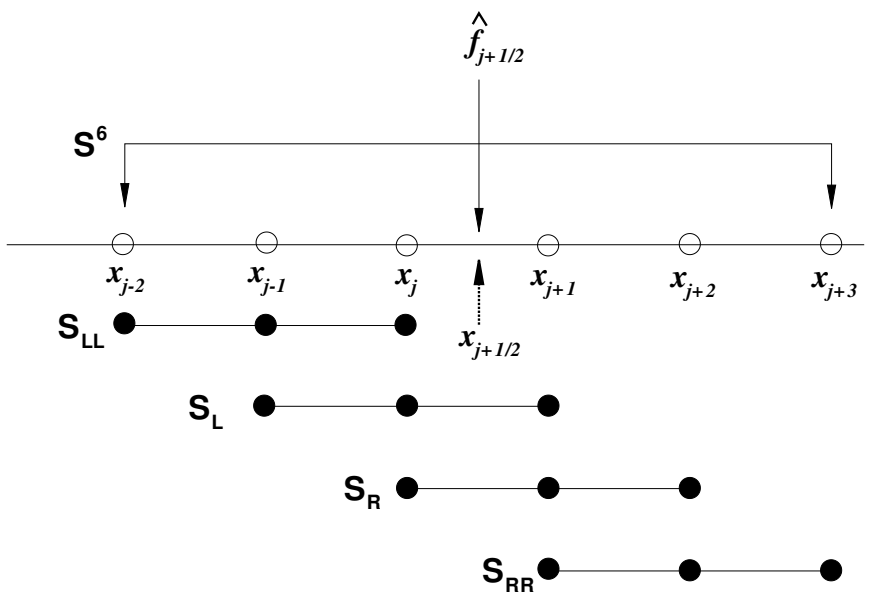

Figure 1: Extended six-point stencil $S^{6}$, and corresponding candidate stencils $S_{L L}, S_{L}, S_{R}$, and $S_{R R}$ for one-parameter family of fifth-order WENO schemes.

in the upwind direction. A central WENO scheme can be constructed from the conventional fifth-order WENO scheme by including an additional downwind candidate stencil $S_{R R}=\left\{x_{j+1}, x_{j+2}, x_{j+3}\right\}$, so that the collection of all four stencils is symmetric with respect to point $\left(j+\frac{1}{2}\right)$. ${ }^{1}$ The WENO flux, constructed in this manner, is given by

$$
\hat{f}_{j+\frac{1}{2}}=w_{j+1 / 2}^{L L} f_{j+1 / 2}^{L L}+w_{j+1 / 2}^{L} f_{j+1 / 2}^{L}+w_{j+1 / 2}^{R} f_{j+1 / 2}^{R}+w_{j+1 / 2}^{R R} f_{j+1 / 2}^{R R},
$$

where $f_{j+1 / 2}^{(r)}, r=\{L L, L, R, R R\}$ are third-order fluxes defined on these four stencils:

$$
\left(\begin{array}{c}
f^{L L}\left(u_{j+1 / 2}\right) \\
f^{L}\left(u_{j+1 / 2}\right) \\
f^{R}\left(u_{j+1 / 2}\right) \\
f^{R R}\left(u_{j+1 / 2}\right)
\end{array}\right)=\frac{1}{6}\left(\begin{array}{cccccc}
2 & -7 & 11 & & & 0 \\
& -1 & 5 & 2 & & \\
& & 2 & 5 & -1 & \\
0 & & & 11 & -7 & 2
\end{array}\right)\left(\begin{array}{c}
f\left(u_{j-2}\right) \\
f\left(u_{j-1}\right) \\
f\left(u_{j}\right) \\
f\left(u_{j+1}\right) \\
f\left(u_{j+2}\right) \\
f\left(u_{j+3}\right)
\end{array}\right)
$$

and $w^{L L}, w^{L}, w^{R}$, and $w^{R R}$ are weight functions that are assigned to the four stencils $S_{L L}, S_{L}, S_{R}$, and $S_{R R}$, respectively. The terms $w^{L L}, w^{L}, w^{R}$, and $w^{R R}$ in equation (14) are nonlinear weight functions. These have both preferred values that are derived from an underlying linear scheme as well as solutiondependent components. The preferred values are given by

$$
d^{L L}=\frac{1}{10}-\varphi ; \quad d^{L}=\frac{6}{10}-3 \varphi ; \quad d^{R}=\frac{3}{10}+3 \varphi ; \quad d^{R R}=\varphi ，
$$

where $\varphi$ is a parameter. The convergence rate of the scheme [eqs. (13)-(16)] with the preferred values $w^{(r)}=d^{(r)}$ and $r=\{L L, L, R, R R\}$ is greater than or equal to 5 for all values of the parameter $\varphi$ in equation (16). For the specific value $\varphi_{c}=\frac{1}{20}$, the fifth-order term vanishes and the convergence rate

\footnotetext{
${ }^{1}$ The above approach to constructing central WENO schemes is proposed in reference [6]. In general, central high-order WENO schemes that are built in this manner, are unstable when unresolved features or strong discontinuities exist in the computational domain. The generalized energy-stable methodology presented in the next section guarantees the stability of the even order approximations while maintaining their nonoscillatory properties.
} 
increases to 6. The classical fifth-order upwind-biased WENO scheme of Jiang and Shu is obtained for $\varphi=0$.

The weight functions $w^{L L}, w^{L}, w^{R}$, and $w^{R R}$ needed in equation (14) are given by

$$
w_{j+\frac{1}{2}}^{(r)}=\frac{\alpha_{r}}{\sum_{l} \alpha_{l}}
$$

where

$$
\alpha_{r}=d^{(r)}\left(1+\frac{\tau_{p}}{\epsilon+\beta^{(r)}}\right), r=\{L L, L, R, R R\}
$$

and $\epsilon \leq O\left(\Delta x^{2}\right)$. The functions $\beta^{(r)}$ are the classical smoothness indicators. [See equation (59) for the general expression.] For fifth-order schemes, they are given by

$$
\begin{aligned}
& \beta^{L L}=\frac{13}{12}\left(u_{j-2}-2 u_{j-1}+u_{j}\right)^{2}+\frac{1}{4}\left(u_{j-2}-4 u_{j-1}+3 u_{j}\right)^{2} \\
& \beta^{L}=\frac{13}{12}\left(u_{j-1}-2 u_{j}+u_{j+1}\right)^{2}+\frac{1}{4}\left(u_{j-1}-u_{j+1}\right)^{2} \\
& \beta^{R}=\frac{13}{12}\left(u_{j}-2 u_{j+1}+u_{j+2}\right)^{2}+\frac{1}{4}\left(3 u_{j}-4 u_{j+1}+u_{j+2}\right)^{2} \\
& \beta^{R R}=\frac{13}{12}\left(u_{j+1}-2 u_{j+2}+u_{j+3}\right)^{2}+\frac{1}{4}\left(-5 u_{j+1}+8 u_{j+2}-3 u_{j+3}\right)^{2} .
\end{aligned}
$$

The $\tau_{p}$ also varies with discretization order; the expression for $\tau_{5}$ is given by

$$
\tau_{5}= \begin{cases}\left(-f_{j-2}+5 f_{j-1}-10 f_{j}+10 f_{j+1}-5 f_{j+2}+f_{j+3}\right)^{2} & , \text { for } \varphi \neq 0 \\ \left(f_{j-2}-4 f_{j-1}+6 f_{j}-4 f_{j+1}+f_{j+2}\right)^{2}, & \text { for } \varphi=0\end{cases}
$$

Expressions for the fourth-, seventh-, and eighth-order WENO schemes appear in the appendix.

The WENO mechanics expressed in equations (17)-(20) represents a significant departure from the mechanics used in the original algorithm by Jiang and Shu [2]. For example, equation (18) replaces the expression

$$
\alpha_{r}=\frac{d^{(r)}}{\left(\epsilon+\beta^{(r)}\right)^{2}}
$$

that is used in the weight functions given in reference [2]. Furthermore, $\epsilon \leq O\left(\Delta x^{2}\right)$ replaces $\epsilon=O\left(10^{-6}\right)$ in [2]. Equations (17)-(20) more closely resemble the weight and smoothness indicators proposed by Borges et al. in [8] There are differences, however, in how the parameters $\tau_{p}$ and $\epsilon$ are chosen; differences motivated by the following observations.

Borges et al.[8] proposes the following function $\tilde{\tau}_{5}$ :

$$
\tilde{\tau}_{5}=\left|\beta^{L L}-\beta^{R}\right|
$$

and a fixed value for the parameter $\epsilon$. Although the above weights and smoothness indicators [eqs. (17)-(19)] with the value of $\tilde{\tau}_{5}$ given by equation (22) significantly outperform the conventional weights of Jiang and Shu [2] for both continuous and discontinuous solutions, three remaining shortcomings include:

1. The value of $\tilde{\tau}_{5}$ given by equation (22) is not smooth because an absolute value function is used, which may reduce accuracy at points where $\beta^{L L}-\beta^{R}$ changes sign.

2. This approach currently does not generalize to WENO schemes with design orders other than 5 . For example, neither $\tilde{\tau}_{6}=\left|\beta^{L L}-\beta^{R}\right|$ nor $\tilde{\tau}_{6}=\left|\beta^{L L}-\beta^{R R}\right|$ provides the design order of accuracy for the central sixth-order WENO scheme.

3. Near critical points where $f^{\prime}, f^{\prime \prime}$, and $f^{\prime \prime \prime}$ vanish simultaneously, the modified weights [eqs. (17)(19), and (22)] fail to provide the design order of convergence for the fifth-order WENO scheme if no constraints are imposed on the parameter $\epsilon$ other than $\epsilon>0$. An example that demonstrates this property is presented in Section 6.1. 
Selecting $\tau_{5}$ in equation (20) to be a quadratic function of the fifth-degree undivided difference defined on the entire six-point stencil, circumvents the first, and second drawbacks encountered when using the scheme suggested in reference [8]. Indeed, $\tau_{5}$ is a $C^{\infty}$ function in its arguments, and can readily be generalized to WENO schemes of order $p$ by choosing $\tau_{p}$ to be the $p$ th-degree undivided difference that is defined on the entire $(p+1)$-point stencil. In contrast to the $\tilde{\tau}_{5}$ found in reference [8], which is of order $O\left(\Delta x^{5}\right)$ for smooth solutions, the proposed function $\tau_{5}$ as given by equation (20) is of order $O\left(\Delta x^{8}\right)$ and $O\left(\Delta x^{10}\right)$ for $\varphi=0$ and $\varphi \neq 0$, respectively; thus much faster convergence of the fifth- and sixth-order WENO schemes to the corresponding underlying linear schemes is achieved. ${ }^{2}$

A remedy for the third shortcoming requires an additional modification to the approach proposed in reference [8]. Both the fifth- and sixth-order WENO schemes with the new weights that are given by equations (17-20) are design-order accurate for smooth solutions, including points at which the firstand second-order derivatives of the solution vanish simultaneously. However, if all derivatives up to fourth are equal to zero, then the fifth- and sixth-order WENO schemes locally become only third-order accurate. To fully resolve this issue, the constraint $\epsilon \leq O\left(\Delta x^{2}\right)$ is imposed herein.

Equations (13)-(20) describe a new family of fifth-order WENO schemes. The primary difference between existing WENO schemes and this new family, is in the stencil biasing mechanics described by equations (17)-(20). Detailed theoretical justifications for the choice of $\tau_{p}$ and $\epsilon$ used in equations (17)(20) are presented in sections 5.2, 5.3. There, and again in the results section, it is shown that these parameters provide fast convergence of the new WENO and ESWENO schemes to the corresponding underlying linear schemes for smooth solutions, and deliver improved shock-capturing capabilities near unresolved features.

\section{A General Approach to Constructing High-Order Energy- Stable Schemes}

Algorithm (1) transforms an existing WENO scheme into an ESWENO scheme that is characterized by the following four properties: 1) a bounded energy estimate for arbitrary nonsmooth initial data, 2) conservation, 3) design order accuracy for sufficiently smooth data, and 4) discontinuity (shock) capturing capabilities that are similar to those of the base WENO scheme.

By construction, algorithm (1) is applicable to 1-D periodic schemes of any order and produces a modified scheme that automatically satisfies the first and second properties: bounded energy estimate and conservation (See reference [1] for details.) Likewise, as shown in the next section, the additional terms added in the ESWENO formulation do not degrade the formal accuracy of the original WENO discretization (property three). However, the degree to which the two formulations differ for unresolved data is not clear. Thus, the properties of the new ESWENO scheme must be tested to ensure it retains the desirable properties of the original formulation.

\section{ESWENO Schemes of Fifth- and Sixth-order}

We now apply algorithm (1) to the one-parameter family of fifth-order WENO schemes [eqs. (17)-(20)] for the scalar 1-D wave equation (1) with $a \geq 0$. Combining equation (15) with equation (14) and substituting the resulting WENO flux $\hat{f}_{j+\frac{1}{2}}$ into equation (13) produces a stencil for the $j$ th grid point

\footnotetext{
${ }^{2}$ The conventional fifth-order WENO scheme that corresponds to $\varphi=0$, does not include the downwind stencil $S_{R R}$; therefore, the fourth-degree undivided difference built on the available five-point stencil, is included in equation (20). This approach to handle the narrow stencil encountered with $\varphi=0$, also generalizes to other orders of accuracy.
} 


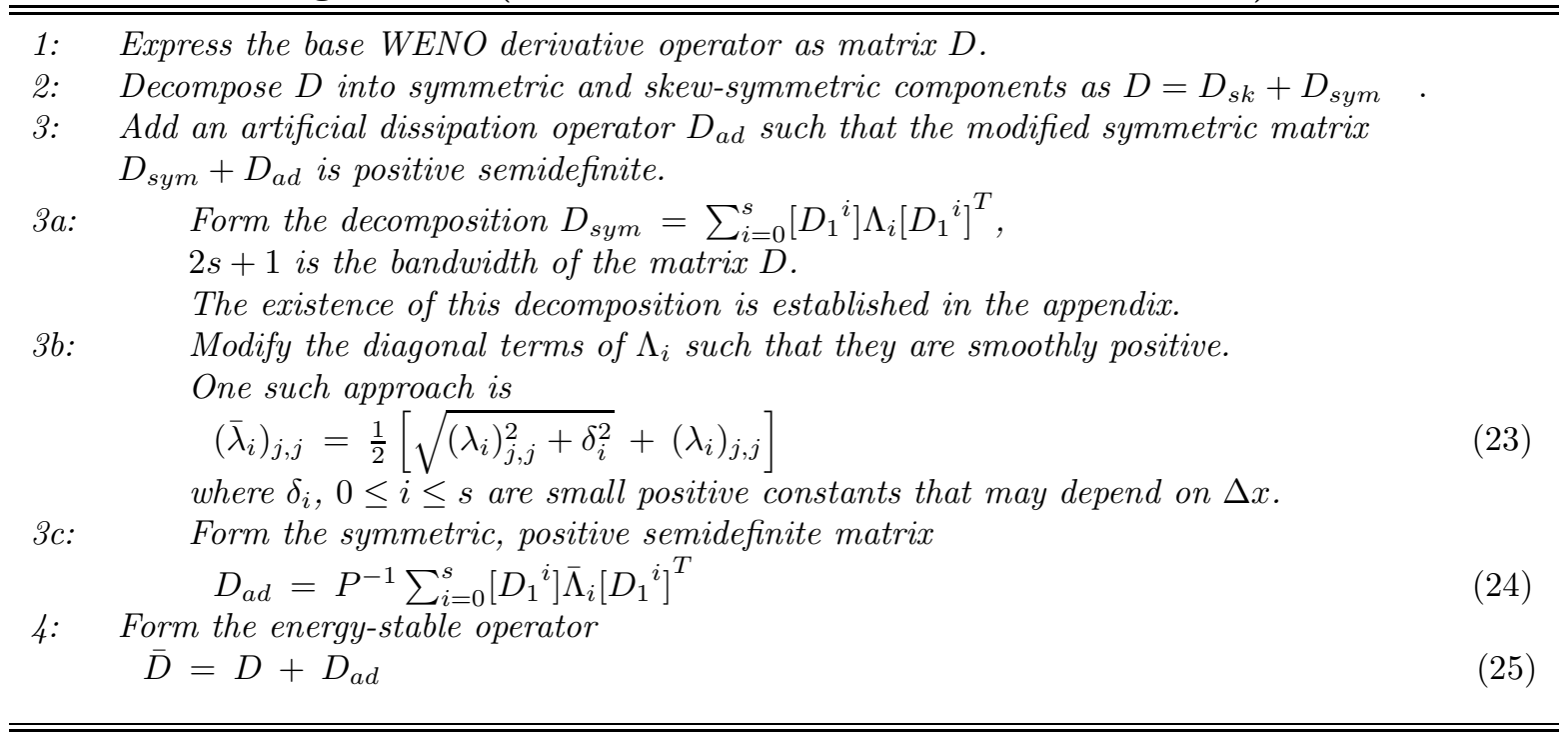

of the form

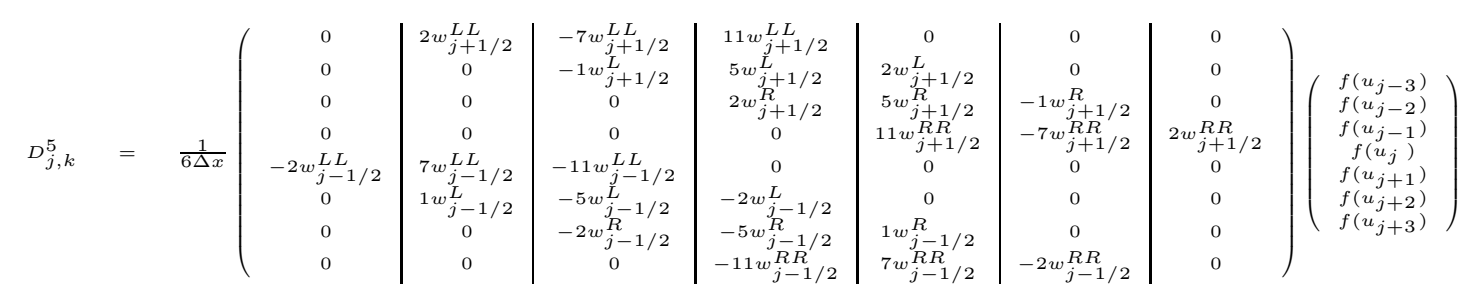

with $k$ on the interval $j-3 \leq k \leq j+3$. An explicit expression for the differentiation matrix $D^{5}$ follows immediately from equation (26).

The derivative matrix $D^{5}$ is now decomposed into symmetric and skew-symmetric parts as

$$
D^{5}=D_{\text {skew }}^{5}+D_{\text {sym }}^{5} .
$$

As with the third-order case (ref. [1]), the skew-symmetric component of $D^{5}$ and the norm, take the forms

$$
D_{\text {skew }}^{5}=P^{-1} Q_{5} \quad ; \quad Q_{5}+Q_{5}^{T}=0 \quad ; \quad P=\Delta x I .
$$

while the matrix $D_{\text {sym }}^{5}$ is expressed as

$$
D_{\text {sym }}^{5}=P^{-1}\left(D_{1}^{3} \Lambda_{3}^{5}\left[D_{1}^{3}\right]^{T}+D_{1}^{2} \Lambda_{2}^{5}\left[D_{1}^{2}\right]^{T}+D_{1}^{1} \Lambda_{1}^{5}\left[D_{1}^{1}\right]^{T}+D_{1}^{0} \Lambda_{0}^{5}\left[D_{1}^{0}\right]^{T}\right) .
$$

The matrices $\Lambda_{j}^{5}$ are diagonal with expressions for the $j$ th element defined by

$$
\begin{aligned}
& \left(\lambda_{3}^{5}\right)_{j, j}=\frac{1}{6}\left[w_{j+5 / 2}^{L L}-w_{j+1 / 2}^{R R}\right] \\
& \left(\lambda_{2}^{5}\right)_{j, j}=\frac{1}{12}\left[\begin{array}{c}
+w_{j+3 / 2}^{L L}-4 w_{j+5 / 2}^{L L}+w_{j+3 / 2}^{L} \\
-w_{j+1 / 2}^{R}+4 w_{j-1 / 2}^{R R}-w_{j+1 / 2}^{R R}
\end{array}\right] \\
& \left(\lambda_{1}^{5}\right)_{j, j}=\frac{1}{12}\left[\begin{array}{ccccc} 
& & 3 w_{j+1 / 2}^{L L} & -5 w_{j+3 / 2}^{L L} & +2 w_{j+5 / 2}^{L L} \\
& & +w_{j+1 / 2}^{L} & -w_{j+3 / 2}^{L} & \\
& +w_{j-1 / 2}^{R} & -w_{j+1 / 2}^{R} & \\
-2 w_{j-3 / 2}^{R R} & +5 w_{j-1 / 2}^{R R} & -3 w_{j+1 / 2}^{R R} &
\end{array}\right]
\end{aligned}
$$




$$
\left(\lambda_{0}^{5}\right)_{j, j}=\frac{1}{2}\left[\begin{array}{c}
-w_{j-1 / 2}^{L L}-w_{j-1 / 2}^{L}-w_{j-1 / 2}^{R}-w_{j-1 / 2}^{R R} \\
+w_{j+1 / 2}^{L L}+w_{j+1 / 2}^{L}+w_{j+1 / 2}^{R}+w_{j+1 / 2}^{R R}
\end{array}\right]=0
$$

Because $\sum_{r} w_{:}^{(r)}=1, \Lambda_{0}^{5}$ is always equal to zero, and is not included in $D_{a d}^{5}$. The sign of the diagonal terms $\left(\lambda_{i}{ }^{5}\right)_{j, j}, i=1,2,3$, could be either positive or negative; thus, the conventional fifth- and sixthorder WENO schemes may become locally unstable. If we define $\left(\bar{\lambda}_{i}^{5}\right)_{j, j}, i=1,2,3$, to be smoothly positive

$$
\left(\bar{\lambda}_{i}^{5}\right)_{j, j}=\frac{1}{2}\left[\sqrt{\left(\lambda_{i}^{5}\right)_{j, j}^{2}+\delta_{i}^{2}}+\left(\lambda_{i}^{5}\right)_{j, j}\right]
$$

then the additional artificial dissipation operator becomes $\bar{D}_{a d}^{5}=P^{-1} \sum_{i=1}^{s}\left[D_{1}^{i}\right]\left(\bar{\Lambda}_{i}^{5}\right)\left[D_{1}{ }^{i}\right]^{T}$, and the resulting energy-stable scheme is obtained by adding the additional dissipation term to the original WENO scheme. That is,

$$
\bar{D}^{5}=D^{5}+\bar{D}_{a d}^{5} .
$$

By construction, $D^{5}$ satisfies all of the conditions of Theorem 1 , thereby providing stability of the fifthand sixth-order ESWENO schemes that are defined by equation (32).

\section{Consistency Analysis}

\subsection{Necessary and Sufficient Conditions for Consistency of WENO Schemes}

We now derive the necessary and sufficient conditions for the weight functions $w^{(r)}$ for a family of $p$ thorder WENO schemes to attain the design order of accuracy. Similar conditions have been obtained for the conventional fifth-order WENO scheme in reference [7]. With the same approach discussed in section 3 for $p=5$, a one-parameter family of $p$ th-order WENO fluxes can be constructed by using a convex combination of $(s+1)$ fluxes $f_{:}^{(r)}$ of $s$ th order as

$$
\hat{f}_{j \pm 1 / 2}=\sum_{r} w_{j \pm 1 / 2}^{(r)} f_{j \pm 1 / 2}^{(r)}
$$

with

$$
f_{j \pm 1 / 2}^{(r)}=h\left(x_{j \pm 1 / 2}\right)+\sum_{l=s}^{p} c_{l}^{(r)} \Delta x^{l}+O\left(\Delta x^{p+1}\right),
$$

where $h(x)$ is the numerical flux function that is implicitly defined as

$$
f(x)=\frac{1}{\Delta x} \int_{x-\frac{\Delta x}{2}}^{x+\frac{\Delta x}{2}} h(\eta) d \eta
$$

and $c_{l}^{(r)}$ are constants that do not depend on $\Delta x$.

The corresponding $p$ th-order WENO operator that approximates the first-order spatial derivative is given by

$$
\left[D^{p} \mathbf{f}\right]_{j}=\frac{\hat{f}_{j+1 / 2}-\hat{f}_{j-1 / 2}}{\Delta x}=\frac{\sum_{r}\left(w_{j+1 / 2}^{(r)} f_{j+1 / 2}^{(r)}-w_{j-1 / 2}^{(r)} f_{j-1 / 2}^{(r)}\right)}{\Delta x}
$$

where $[\cdot]_{j}$ is a $j$ th component of a vector, the index $r$ in equation $(36)$ sweeps over all $(s+1)$ stencils, and $w^{(r)}$ is a nonlinear weight function that is assigned to the corresponding $s$-point stencil $S_{r}$. For sufficiently smooth solutions, the weights $w_{:}^{(r)}$ approach their preferred values $d^{(r)}$, so that the WENO operator converges to the target linear operator $D^{\text {Target }}$ as

$$
\left[D^{\text {Target }} \mathbf{f}\right]_{j}=\frac{f_{j+1 / 2}^{\text {Target }}-f_{j-1 / 2}^{\text {Target }}}{\Delta x}=\frac{\sum_{r}\left(d^{(r)} f_{j+1 / 2}^{(r)}-d^{(r)} f_{j-1 / 2}^{(r)}\right)}{\Delta x},
$$


where $d^{(r)}$ is a one-parameter family of constants chosen to ensure $p$ th-order convergence of the target operator to the exact value of the first-order derivative at $x_{j}$. That is,

$$
\left[D^{\text {Target }} \mathbf{f}\right]_{j}=\left.\frac{\partial f}{\partial x}\right|_{x=x_{j}}+O\left(\Delta x^{p}\right)
$$

The coefficients $d^{(r)}$ for one-parameter families of linear target schemes up to eighth order are given in the appendix. All target linear schemes in the family are $(2 s-1)$ th-order accurate, except one central scheme, which is $(2 s)$ th-order accurate.

Subtracting equation (37) from equation (36) and using equation (34), we have

$$
\begin{aligned}
& {\left[D^{p} \mathbf{f}\right]_{j}-\left[D^{\text {Target }} \mathbf{f}\right]_{j}=\frac{\sum_{r}\left[\left(w_{j+1 / 2}^{(r)}-d^{(r)}\right) f_{j+1 / 2}^{(r)}-\left(w_{j-1 / 2}^{(r)}-d^{(r)}\right) f_{j-1 / 2}^{(r)}\right]}{\Delta x}} \\
& =\frac{1}{\Delta x} \sum_{r}\left[\left(w_{j+1 / 2}^{(r)}-d^{(r)}\right) h^{(r)}\left(x_{j+1 / 2}\right)-\left(w_{j-1 / 2}^{(r)}-d^{(r)}\right) h^{(r)}\left(x_{j-1 / 2}\right)\right] \\
& +\sum_{l=s}^{p} \sum_{r} \Delta x^{l-1} c_{l}^{(r)}\left[\left(w_{j+1 / 2}^{(r)}-d^{(r)}\right)-\left(w_{j-1 / 2}^{(r)}-d^{(r)}\right)\right]+O\left(\Delta x^{p}\right)
\end{aligned}
$$

From equation (39) it immediately follows that to retain $p$ th-order accuracy, the weights of the WENO operator $D^{p}$ should satisfy the following necessary and sufficient conditions:

$$
\begin{aligned}
& \sum_{r}\left[w_{j \pm 1 / 2}^{(r)}-d^{(r)}\right]=O\left(\Delta x^{p+1}\right) \\
& \sum_{r} c_{s}^{(r)}\left[\left(w_{j+1 / 2}^{(r)}-d^{(r)}\right)-\left(w_{j-1 / 2}^{(r)}-d^{(r)}\right)\right]=O\left(\Delta x^{p-s+1}\right) \\
& \cdots \\
& \sum_{r} c_{p}^{(r)}\left[\left(w_{j+1 / 2}^{(r)}-d^{(r)}\right)-\left(w_{j-1 / 2}^{(r)}-d^{(r)}\right)\right]=O(\Delta x)
\end{aligned}
$$

Here, we use the following properties of $h(x)$ and $d^{(r)}: f^{\prime}(x)=\frac{h\left(x_{j+1 / 2}\right)-h\left(x_{j-1 / 2}\right)}{\Delta x}$, and $\sum_{r} d^{(r)}=1$.

By construction, the weights [eq. (17)] are normalized such that $\sum_{r} w_{:}^{(r)}=1$; thus, the first constraint in equation (40) is satisfied identically. To simplify the analysis, especially for $f(x)$ with an arbitrary number of vanishing derivatives, we hereafter use the following sufficient condition on $w^{(r)}$ for the conventional WENO scheme to attain $p$ th-order accuracy:

$$
w_{:}^{(r)}-d^{(r)}=O\left(\Delta x^{p-s+1}\right) .
$$

This constraint is a direct consequence of the necessary and sufficient conditions [eq. (40)].

\subsection{Sufficient Conditions for Consistency of ESWENO Schemes}

In this section, we show that the conditions [eq. (41)] and the constraints on $\delta_{i}$ in equation (23):

$$
\delta_{i}=O\left(\Delta x^{p-2 i+1}\right), \quad 1 \leq i \leq s
$$

guarantee that the energy-stable modifications of the conventional $p$ th-order WENO scheme (see section 4) preserve the design order of the original scheme. As follows from equation (25), the ESWENO operator consists of two terms: one is the original WENO operator and the other is the additional artificial dissipation operator $D_{a d}$ that is given by equation (24). As shown in the previous section, the WENO operator is $p$ th-order accurate if equation (41) holds. Hence, we need only show that $D_{a d} \mathbf{f}=O\left(\Delta x^{p}\right)$.

Let us prove this conjecture for the one-parameter family of fifth-order ESWENO schemes that is presented in Section 4.1. Note that the term $P^{-1} D_{1}^{3} \Lambda_{0}\left[D_{1}^{3}\right]^{T}$ in equation (27) is identically equal to zero because of the normalization $\sum_{r} w_{:}^{(r)}=1$; it need not be included in $D_{a d}$. Thus, the additional artificial dissipation term is given by

$$
D_{a d} \mathbf{f}=P^{-1} D_{1} \bar{\Lambda}_{1}\left[D_{1}\right]^{T} \mathbf{f}+P^{-1} D_{1}^{2} \bar{\Lambda}_{2}\left[D_{1}^{2}\right]^{T} \mathbf{f}+P^{-1} D_{1}^{3} \bar{\Lambda}_{3}\left[D_{1}^{3}\right]^{T} \mathbf{f}
$$




$$
\bar{\lambda}_{i}=\frac{1}{2}\left[\sqrt{\lambda_{i}{ }^{2}+\delta_{i}^{2}}+\lambda_{i}\right], \quad i=1,2,3
$$

where $\lambda_{i}$ is a $j$ th diagonal element of the matrix $\Lambda_{i}$. For simplicity, we have omitted the superscript 5 and the subscript $(j, j)$ in this section.

First, we evaluate the terms $\lambda_{1}, \lambda_{2}$, and $\lambda_{3}$ that are defined by equations (28-30). To simplify the derivation, the sufficient conditions [eq. (41)] for the one-parameter family of fifth-order ESWENO schemes is rewritten in the following form:

$$
w_{:}^{(r)}-d^{(r)}=\left(\varphi-\frac{1}{20}\right) O\left(\Delta x^{3}\right)+O\left(\Delta x^{4}\right) .
$$

In equation (45), the stencil width $s$ of each reconstruction polynomial is equal to 3 , and the order $p$ of this family of schemes is equal to 5 if $\varphi \neq \frac{1}{20}$ or 6 if $\varphi=\frac{1}{20}$. As follows from equation (45), the stiffer constraint on the weights should be imposed to obtain sixth-order accuracy.

Replacing $w_{:}^{(r)}$ in $\lambda_{2}$ with the corresponding preferred values $d^{(r)}$ and using equations (16) and (29), for any value of the parameter $\varphi$ yields

$$
\frac{1}{12}\left[d^{L L}-4 d^{L L}+d^{L}-d^{R}+4 d^{R R}-d^{R R}\right] \equiv 0
$$

Subtracting equation (46) from equation (29) and taking into account equation (45) yields

$$
\begin{aligned}
\lambda_{2}= & \frac{1}{12}\left[\left(w_{j+3 / 2}^{L L}-d^{L L}\right)-4\left(w_{j+5 / 2}^{L L}-d^{L L}\right)+\left(w_{j+3 / 2}^{L}-d^{L}\right)\right. \\
& \left.-\left(w_{j+1 / 2}^{R}-d^{R}\right)+4\left(w_{j-1 / 2}^{R R}-d^{R R}\right)-\left(w_{j+1 / 2}^{R R}-d^{R R}\right)\right] \\
= & \left(\varphi-\frac{1}{20}\right) O\left(\Delta x^{3}\right)+O\left(\Delta x^{4}\right) .
\end{aligned}
$$

By comparing equations (29) and (30), one can see that $\lambda_{1}$ is at least one order higher than that of $\lambda_{2}$ because of an additional cancellation that occurs within each group of terms that is associated with the same stencil. For example, expanding all of the terms $w_{:}^{L L}$ in equation (30) about $x_{j}$ yields

$$
3 w_{j+1 / 2}^{L L}-5 w_{j+3 / 2}^{L L}+2 w_{j+5 / 2}^{L L}=-\left.2 \frac{\partial w^{L L}}{\partial x}\right|_{x_{j}} \Delta x+O\left(\Delta x^{2}\right),
$$

which gives an extra factor $O(\Delta x)$ compared with the corresponding terms $w_{:}^{L L}$ in equation (30): $w_{j+3 / 2}^{L L}-4 w_{j+5 / 2}^{L L}=O(1)$. The same conclusion can be drawn for the other groups of terms that are associated with the $L, R$, and $R R$ stencils in equation (30), which leads to

$$
\lambda_{1}=\left(\varphi-\frac{1}{20}\right) O\left(\Delta x^{4}\right)+O\left(\Delta x^{5}\right) .
$$

Applying the same procedure to $\lambda_{3}$ yields

$$
\begin{aligned}
\lambda_{3} & =\frac{1}{6}\left(d^{L L}-d^{R R}\right)+\frac{1}{6}\left[\left(w_{j+5 / 2}^{L L}-d^{L L}\right)-\left(w_{j+1 / 2}^{R R}-d^{R R}\right)\right] \\
& =\frac{1}{6}\left(\frac{1}{10}-2 \varphi\right)+\left(\varphi-\frac{1}{20}\right) O\left(\Delta x^{3}\right)+O\left(\Delta x^{4}\right) .
\end{aligned}
$$

To evaluate each term in equation (43), we consider three cases: 1) $\left.\left|\lambda_{i}\right| \gg \delta_{i}>0,2\right) \lambda_{i}=O\left(\delta_{i}\right)$, and 3) $\left|\lambda_{i}\right| \ll \delta_{i}, i=1,2,3$. If we assume that $\left|\lambda_{i}\right| \gg \delta_{i}>0$, then equation (44) can be expanded as follows:

$$
\bar{\lambda}_{i}=\frac{\left|\lambda_{i}\right|+\lambda_{i}}{2}+\frac{\delta_{i}^{2}}{4\left|\lambda_{i}\right|},
$$

which yields

$$
\bar{\lambda}_{i}=\left\{\begin{array}{rl}
\lambda_{i}, & \text { if } \lambda_{i}>0 \\
\delta_{i}, & \text { if } \lambda_{i}=0 \\
\frac{\delta_{i}^{2}}{4\left|\lambda_{i}\right|}, & \text { if } \lambda_{i}<0
\end{array},\right.
$$


where the higher order terms have been omitted. Because equation (52) has been derived under the assumption that $\left|\lambda_{i}\right| \gg \delta_{i}>0$, we can immediately conclude that $\left|\lambda_{i}\right| \gg \delta_{i} \gg \frac{\delta_{i}^{2}}{4\left|\lambda_{i}\right|}$. Therefore, we only need consider that

$$
\bar{\lambda}_{i}=\lambda_{i},
$$

which provides the lowest order of convergence for the term $D_{i}^{i} \bar{\Lambda}_{i}\left[D_{1}^{i}\right]^{T} \mathbf{f}$. If we substitute equation (53) in equation (43) and use equations $(47,49,50)$, then the additional ESWENO dissipation term becomes

$$
\begin{aligned}
D_{a d} \mathbf{f} & =\left[\left(\varphi-\frac{1}{20}\right) O\left(\Delta x^{3}\right)+O\left(\Delta x^{4}\right)\right] D_{1}\left[D_{1}\right]^{T} \mathbf{f} \\
& +\left[\left(\varphi-\frac{1}{20}\right) O\left(\Delta x^{2}\right)+O\left(\Delta x^{3}\right)\right] D_{1}^{2}\left[D_{1}^{2}\right]^{T} \mathbf{f} \\
& +\left[\frac{1}{10}-2 \varphi\right. \\
6 \Delta x & \left.\left(\varphi-\frac{1}{20}\right) O\left(\Delta x^{2}\right)+O\left(\Delta x^{3}\right)\right] D_{1}^{3}\left[D_{1}^{3}\right]^{T} \mathbf{f}
\end{aligned}
$$

If we take into account that $D_{1}^{i}\left[D_{1}^{i}\right]^{T} \mathbf{f}=O\left(\Delta x^{2 i}\right)$, then $D_{a d} \mathbf{f}$ can be recast as

$$
D_{a d} \mathbf{f}=\left(\varphi-\frac{1}{20}\right) O\left(\Delta x^{5}\right)+O\left(\Delta x^{6}\right)
$$

From equation (55) we see, that the additional dissipation term is at least fifth-order accurate for all values of the parameter $\varphi$. The fifth-order term vanishes for $\varphi=\frac{1}{20}$; for this value the order increase by one to sixth-order.

The second case can be considered in a similar manner. Substituting $\lambda_{i}=O\left(\delta_{i}\right), i=1,2,3$, in equation (43) yields

$$
\begin{aligned}
D_{a d} \mathbf{f} & =\frac{O\left(\delta_{1}\right)}{\Delta x} D_{1}\left[D_{1}\right]^{T} \mathbf{f}+\frac{O\left(\delta_{2}\right)}{\Delta x} D_{1}^{2}\left[D_{1}^{2}\right]^{T} \mathbf{f}+\frac{O\left(\delta_{3}\right)}{\Delta x} D_{1}^{3}\left[D_{1}^{3}\right]^{T} \mathbf{f} \\
& =O\left(\delta_{1} \Delta x\right)+O\left(\delta_{2} \Delta x^{3}\right)+O\left(\delta_{3} \Delta x^{5}\right)
\end{aligned}
$$

To guarantee that the ESWENO dissipation term is $p$ th-order accurate, the following constraints should be imposed on $\delta_{i}$ :

$$
\begin{aligned}
& \delta_{1}=O\left(\Delta x^{p-1}\right) \\
& \delta_{2}=O\left(\Delta x^{p-3}\right) \\
& \delta_{3}=O\left(\Delta x^{p-5}\right),
\end{aligned}
$$

where $p$ is equal to 5 for $\varphi \neq \frac{1}{20}$ or 6 for $\varphi=\frac{1}{20}$. Note that the constraints [eq. (57)] are fully consistent with those that are given by equation (42). The third case, $\left|\lambda_{i}\right| \ll \delta_{i}$, is similar to the second one and results in the same constraints [eq. (57)] on $\delta_{i}$; therefore the third case is not presented here.

Remark 4 . Note that $\delta_{i}, i=1,2,3$, are user-defined parameters; therefore, the conditions [eq. (42)] can always be met.

Remark 5. Although only fifth- and sixth-order ESWENO schemes have been analyzed in this section, the same procedure is directly applicable to the additional ESWENO schemes presented in the appendix. Thus, we can conclude that if equations (41) and (42) hold, then all the ESWENO schemes that are considered in this paper are design-order accurate.

\subsection{Consistency of the ESWENO scheme with New Weights}

The new weight functions for the one-parameter family of $p$ th-order WENO and ESWENO schemes are given by

$$
w^{(r)}=\frac{\alpha_{r}}{\sum_{l} \alpha_{l}}, \quad \alpha_{r}=d^{(r)}\left(1+\frac{\tau_{p}}{\epsilon+\beta^{(r)}}\right),
$$

where $\beta^{(r)}$ are the classical smoothness indicators:

$$
\beta^{(r)}=\sum_{l=1}^{s-1} \Delta x^{2 l-1} \int_{x_{j-\frac{1}{2}}}^{x_{j+\frac{1}{2}}}\left(\frac{d^{l} q_{r}(x)}{d^{l} x}\right)^{2} d x,
$$


$q_{r}(x)$ is an $(s-1)$ th-degree reconstruction polynomial that is defined on a stencil $S_{r}$, and $\epsilon$ is a small positive parameter that can depend on $\Delta x$. In equation (58), $\tau_{p}$ is defined by

$$
\begin{gathered}
\tau_{p}=\left(V<x_{j-s+1}, \ldots, x_{j+s}>\right)^{2}, \text { for } \varphi \neq 0 \\
\tau_{p}=\left(V<x_{j-s+1}, \ldots, x_{j+s-1}>\right)^{2}, \text { for } \varphi=0
\end{gathered}
$$

where $V<x_{j-s+1}, \ldots, x_{j+s}>$ is the $p$ th-degree, undivided difference. Note that for the original WENO schemes of Jiang and Shu [2], which correspond to $\varphi=0$, the entire stencil includes only $(2 s-1)$ points; therefore, the highest degree of undivided differences that can be constructed on this stencil is $2 s-2$, rather than $2 s-1$, which is used for the other schemes in this one-parameter family. In particular, $w^{(r)}, \beta^{(r)}$, and $\tau_{5}$ for the fifth- and sixth-order WENO and ESWENO schemes are given by equations (17)-(20). Another scheme that requires special consideration is the central ESWENO scheme, which is obtained by setting $\varphi=\varphi_{c}$, so that it is one order higher than that of the other schemes in the family.

First, we present a truncation error analysis for the entire one-parameter family of $p$ th-order ESWENO schemes, except for the two schemes that correspond to $\varphi=0$ and $\varphi=\varphi_{c}$. We demonstrate that the new weights that are defined by equations (58)-(60) satisfy the sufficient condition [eq. (41)] for smooth solutions with any number of vanishing derivatives if the following constraint is imposed on the parameter $\epsilon$ in equation (58):

$$
\epsilon \geq O\left(\Delta x^{p+s-1}\right)>0
$$

where $p$ is a design order of the scheme and $(s-1)$ is a degree of the corresponding reconstruction polynomials.

If we assume that all of the required derivatives are continuous and use the properties of the Newton divided differences, then the Taylor series expansions of $\beta^{(r)}$ and $\tau_{p}(\varphi \neq 0)$ at $x_{j}$ are given by

$$
\begin{gathered}
\beta^{(r)}={f^{\prime}}^{2} \Delta x^{2}+O\left(\Delta x^{3}\right), \\
\tau_{p}=\left(f^{(p)}\right)^{2} \Delta x^{2 p}+O\left(\Delta x^{2 p+1}\right),
\end{gathered}
$$

where $f^{\prime}$ and $f^{(p)}$ are the first- and $p$ th-order derivatives of $f$ at $x_{j}$. For example, for the family of fifth-order WENO schemes, these expansions are

$$
\begin{gathered}
\beta^{L L}=f^{\prime 2} \Delta x^{2}+\left(\frac{13}{12} f^{\prime \prime 2}-\frac{2}{3} f^{\prime} f^{\prime \prime \prime}\right) \Delta x^{4}+\left(-\frac{13}{6} f^{\prime \prime} f^{\prime \prime \prime}+\frac{1}{2} f^{\prime} f^{\prime \prime \prime \prime}\right) \Delta x^{5}+O\left(\Delta x^{6}\right) \\
\beta^{L}=f^{\prime 2} \Delta x^{2}+\left(\frac{13}{12} f^{\prime \prime 2}+\frac{1}{3} f^{\prime} f^{\prime \prime \prime}\right) \Delta x^{4}+O\left(\Delta x^{6}\right) \\
\beta^{R}=f^{\prime 2} \Delta x^{2}+\left(\frac{13}{12} f^{\prime \prime 2}-\frac{2}{3} f^{\prime} f^{\prime \prime \prime}\right) \Delta x^{4}+\left(\frac{13}{6} f^{\prime \prime} f^{\prime \prime \prime}-\frac{1}{2} f^{\prime} f^{\prime \prime \prime \prime}\right) \Delta x^{5}+O\left(\Delta x^{6}\right) \\
\beta^{R R}=f^{\prime 2} \Delta x^{2}+\left(\frac{13}{12} f^{\prime \prime 2}-\frac{11}{3} f^{\prime} f^{\prime \prime \prime}\right) \Delta x^{4}+\left(\frac{13}{3} f^{\prime \prime} f^{\prime \prime \prime}-5 f^{\prime} f^{\prime \prime \prime \prime}\right) \Delta x^{5}+O\left(\Delta x^{6}\right) \\
\tau_{5}=\left(f^{(5)}\right)^{2} \Delta x^{10}+O\left(\Delta x^{11}\right), \quad \text { for } \varphi \neq 0 .
\end{gathered}
$$

We first consider a case with $f^{\prime}\left(x_{j}\right) \neq 0$. Substituting equations (63) and (64) in equation (58) and accounting for equation (62) yields

$$
\frac{\tau_{p}}{\epsilon+\beta^{(r)}}=O\left(\Delta x^{2 p-2}\right)\left(1-O\left(\Delta x^{p+s-3}\right)\right)
$$

which leads to

$$
w^{(r)}=d^{(r)}+O\left(\Delta x^{2 p-1}\right) .
$$

Note that the order of convergence of $w^{(r)}$ to its preferred value is $2 p-1$ rather than $2 p-2$. The main reason for such "superconvergence" is the additional cancellation that occurs because the leading truncation error terms of all of the smoothness indicators are identical to each other if $f^{\prime} \neq 0$, as can be seen in equation (63). Equations (67) and (68) are valid only for $p \geq 3$ and are not applicable to the third-order WENO and ESWENO schemes that correspond to $\varphi=0$. The detailed analysis of these 
third-order schemes $(\varphi=0)$ is presented in reference [1]. By comparing equation (68) with equation (41), we can immediately conclude that the new weights satisfy the sufficient condition [eq. (41)], ensuring that both the WENO and ESWENO schemes are design-order accurate. Furthermore, for schemes of order $3(\varphi \neq 0)$ or higher, the weights converge to their preferred values at a rate that is significantly faster than that given by the sufficient condition (41). The result is a much faster rate of convergence for both the ESWENO and WENO schemes to the corresponding target linear schemes, even on coarse and moderate grids.

The next issue that is addressed is the convergence of the ESWENO schemes near the critical points at which the first-order and higher order derivatives of the flux approach zero. Let $x_{c}$ be a critical point at which the flux function is sufficiently smooth and at which its derivatives of order up to $n_{v d}$ th are equal to zero. That is,

$$
f^{\prime}\left(x_{c}\right)=\cdots=f^{\left(n_{v d}\right)}\left(x_{c}\right)=0, f^{\left(n_{v d}+1\right)}\left(x_{c}\right) \neq 0 .
$$

In contrast to the previous case for which $f^{\prime} \neq 0$, the leading truncation error terms of the smoothness indicators at the critical point are not equal to each other; thus, no additional cancellation occurs. For any number of vanishing derivatives, the following inequalities always hold:

$$
\epsilon \geq O\left(\Delta x^{p+s-1}\right) \gg O\left(\Delta x^{2 p}\right) \geq \tau_{p}
$$

which yields

$$
\frac{\tau_{p}}{\epsilon+\beta^{(r)}} \ll 1
$$

By using equation (69), the weights can be recast as

$$
w^{(r)}=\frac{d^{(r)}+d^{(r)} \frac{\tau_{p}}{\epsilon+\beta^{(r)}}}{1+\sum_{l} \frac{\tau_{p}}{\epsilon+\beta^{(l)}}}=d^{(r)}+O\left(\frac{\tau_{p}}{\epsilon+\beta^{(r)}}\right),
$$

where we use $\sum_{r} d^{(r)}=1$. For any number of vanishing derivatives, we have

$$
\frac{\tau_{p}}{\epsilon+\beta^{(r)}} \leq \frac{\tau_{p}}{\epsilon} \leq \frac{O\left(\Delta x^{2 p}\right)}{O\left(\Delta x^{p+s-1}\right)}=O\left(\Delta x^{p-s+1}\right) .
$$

From equation (71) we see that $w^{(r)}$ converges to $d^{(r)}$ at the rate of $O\left(\Delta x^{p-s+1}\right)$ or higher and satisfies the sufficient condition (41).

As mentioned at the beginning of this section, the two schemes that correspond to $\varphi=0$ and $\varphi=\varphi_{c}$ require special consideration. Using the same procedure that is outlined above, we can easily show that if the parameter $\epsilon$ satisfies the constraints

$$
\begin{array}{ll}
\epsilon \geq O\left(\Delta x^{3 s-4}\right), & \text { for } \varphi=0 \\
\epsilon \geq O\left(\Delta x^{3 s-3}\right), & \text { for } \varphi=\varphi_{c},
\end{array}
$$

then the corresponding ESWENO schemes are design-order accurate regardless of the number of vanishing derivatives of the solution. The constraints [eq. (72)] are derived using the following relations between the order of the scheme and the degree of the reconstruction polynomials:

$$
\begin{array}{ll}
p=2 s-1, & \text { for } \varphi=0 \\
p=2 s, & \text { for } \varphi=\varphi_{c} .
\end{array}
$$

Also, note that if no constraint is imposed on $\epsilon$ except that it must be strictly positive, then the order of convergence of the $p$ th-order WENO and ESWENO schemes with the new weights may deteriorate from $p$ to $s$. Indeed, for a sufficiently large number of vanishing derivatives $n_{v d}, \beta^{(r)}$ and $\tau_{p}$ may become of the same order. For example, for the family of fifth-order schemes $(\varphi \neq 0)$, this type of degeneration 
occurs at $n_{v d}=4$. If $f^{\prime}=f^{\prime \prime}=f^{\prime \prime \prime}=f^{\prime \prime \prime \prime}=0, f^{(5)} \neq 0$, and $\epsilon \leq O\left(\Delta x^{10}\right)$, which does not satisfy the condition given in equation (62), then equations (65) and (66) lead to

$$
\frac{\tau_{5}}{\epsilon+\beta^{(r)}}=\frac{O\left(\Delta x^{10}\right)}{O\left(\Delta x^{10}\right)+O\left(\Delta x^{10}\right)}=O(1)
$$

As a result, the weights are of order $O(1)$ and the fifth-order WENO and ESWENO schemes locally degenerate to third order.

Remark 6. In reference [8], the following modification of the weight functions given in equations (17)(19), and (22) is proposed to recover the fifth-order rate of convergence if $n_{v d} \leq 2$ :

$$
w^{(r)}=\frac{\alpha_{r}}{\sum_{l} \alpha_{l}}, \quad \alpha_{r}=d^{(r)}\left[1+\left(\frac{\tau_{5}}{\epsilon+\beta^{(r)}}\right)^{m}\right]
$$

where $m=2$. However, the modified weights [eq. (74)] still experience the same degeneration in accuracy near critical points for any choice of the parameter $m$ if $n_{v d} \leq 6$. We demonstrate this with the following "thought" experiment. First, build a polynomial of degree 7 (or higher) such that at least 6 derivatives vanish at a single point. Next, connect this polynomial to a constant polynomial at the point where the derivatives of the first polymonial vanish. The solution to equation (1) is then a six times continuously differentiable function everywhere on the combined piecewise polynomials. Note that the underlying linear scheme is fifth-order accurate in this case. If we assume that the $L L$ stencil is located completely in the constant part of the solution (i.e. $f_{j-2}=f_{j-1}=f_{j}$ ) while the other two stencils include points from the polynomial part of the solution, then we have $\beta^{L L}=0, \beta^{L} \neq 0$, and $\beta^{R} \neq 0$. As a result, $\tau_{5}=\left|\beta^{L L}-\beta^{R}\right|=\beta^{R}$. If no constraint is imposed on $\epsilon$, then we can always choose $\epsilon$ such that $\beta^{R} \gg \epsilon \rightarrow 0$ on any given grid, which yields

$$
w^{(R)}-d^{(R)}=O\left(\frac{\tau_{5}}{\epsilon+\beta^{R}}\right)^{m}=O\left(\frac{\beta^{R}}{\epsilon+\beta^{R}}\right)^{m}=O(1)-O\left(\frac{\epsilon}{\beta^{R}}\right) \rightarrow O(1),
$$

This leads to $w^{R}=O(1)$ and, consequently, to a loss of the design order of accuracy. Note that this problem persists regardless of the choice of $m$ in equation (74).

Remark 7. The parameter $\epsilon$ is user-defined, and therefore, the sufficient conditions [eqs. (62) and (72)] can always be satisfied.

Remark 8. Equations (62) and (72) do not provide sharp estimates for the parameter $\epsilon$, which can be weakened if additional information regarding the number of vanishing derivatives is available a priori. Note, however, that the constraints [eqs. (62) and (72)] guarantee the design order of convergence of the corresponding WENO and ESWENO schemes for smooth solutions with an arbitrary number of vanishing derivatives.

The last issue that we discuss in this section concerns discontinuous and unresolved solutions. To successfully emulate the ENO strategy, a stencil for which the solution is discontinuous is eliminated from the approximation by effectively nullifying the corresponding weight that is associated with this stencil. Let a discontinuity be located inside a stencil $S_{r}$, while the solution is smooth in all other stencils. We can easily verify that $\tau_{p}=O(1)$, because $\tau_{p}$ involves all points of the entire stencil including those that contain the discontinuity. Therefore, the weight $w^{(r)}$ can be evaluated as

$$
w^{(r)}=\frac{d^{(r)}\left[1+\frac{O(1)}{\epsilon+O(1)}\right]}{O(1)+\sum_{l \neq r} \frac{O(1)}{\epsilon+O\left(\Delta x^{2}\right)}}=\frac{O(1)}{O(1)+\frac{O(1)}{\epsilon+O\left(\Delta x^{2}\right)}}=O(1)\left[\epsilon+O\left(\Delta x^{2}\right]\right)
$$

Based on the above equation, to reduce the influence of $\epsilon$ on the solution near the discontinuity, the parameter $\epsilon$ should satisfy the following constraint:

$$
\epsilon \leq O\left(\Delta x^{2}\right)
$$


Indeed, if equation (76) is met, then $w^{(r)}$ is of the order $O\left(\Delta x^{2}\right)$ and has the same order of magnitude as it would have if $\epsilon=0$. Hence, the parameter $\epsilon$ must be bounded not only from below by equations (62) and (72), but also from above by equation (76).

Another consideration that can help us optimally select the parameter $\epsilon$ is the manner in which the WENO and ESWENO schemes handle small-amplitude oscillations. Consider a solution that contains small-amplitude spurious oscillations

$$
f=f_{s}+\delta f
$$

where $f_{s}$ is a smooth component and $\delta f$ is a nonsmooth, high-frequency component of the solution. By substituting equation (77) in equation (59) and assuming that the contribution of the smooth component is negligibly small, we have

$$
\beta^{(r)}=O\left(\delta f^{2}\right) .
$$

As follows from equation (58), if $\epsilon \gg O\left(\delta f^{2}\right)$, then the denominator $\epsilon+\beta^{(r)}$ is dominated by $\epsilon$, and these spurious oscillations cannot be detected by the ESWENO dissipation mechanism. However, if $\epsilon \leq O\left(\delta f^{2}\right)$, then the weights begin to deviate from their preferred values, which increases dissipation in those stencils containing spurious oscillations.

From these considerations it follows that the lower bound of the constraints on $\epsilon$ given by equations (62), (72), and (76) should be used (1) to obtain the design order of convergence at the smooth parts of the solutions, (2) to effectively damp high-frequency spurious oscillations, and (3) to provide good shock-capturing capabilities near strong discontinuities. Therefore, in our numerical experiments, we use

$$
\epsilon= \begin{cases}O\left(\Delta x^{3 s-4}\right), & \text { for } \varphi=0 \\ O\left(\Delta x^{3 s-3}\right), & \text { for } \varphi=\varphi_{c} \\ O\left(\Delta x^{3 s-2}\right), & \text { otherwise }\end{cases}
$$

where $s-1$ is the degree of the reconstruction polynomials. With the above selection of $\epsilon$, all spurious oscillations of amplitude $O\left(\epsilon^{1 / 2}\right)$ and higher are suppressed by the ESWENO dissipation mechanism. The same conclusions can be drawn for the WENO schemes with the new weights given by equations (58)-(61).

\section{$6 \quad$ Numerical Results}

We now assess the performance of the fourth-, fifth-, and sixth-order ESWENO schemes with the new weights and compare them with the conventional WENO counterparts. For all of the numerical experiments that are presented, the parameters $\epsilon$ of the ESWENO weight functions and the parameters $\delta_{i}$ of the additional artificial dissipation operator are chosen based on equations (78) and (42) with two minor modifications. First, to preserve the scale invariance of the original WENO schemes, the physical grid spacing $\Delta x$ in equations (42) and (78) is replaced with $\Delta \xi$, where $\Delta \xi=1 / J$ and $J$ is the total number of grid cells. Second, as follows from equation (58), the parameter $\epsilon$ should be scaled consistently with $\beta^{(r)}$. In regions where the solution is smooth, for the third- and fourth-order WENO and ESWENO schemes, $\beta^{(r)}$ approximates $f^{\prime 2} \Delta \xi^{2}$. For the fifth- and sixth-order schemes, $\beta^{(r)}$ approximates the linear combination of the same first-order derivative term and a second-order derivative term that is proportional to $f^{\prime \prime 2}$. The same pattern persists for higher order schemes as well. In the vicinity of discontinuities, $\beta^{(r)}$ is of the order $O\left(f^{2}\right)$. If we take into account the above considerations, for all test problems considered the parameter $\epsilon$ is set to

$$
\begin{aligned}
& \epsilon= \begin{cases}C \Delta \xi^{3 s-4}, & \text { for } \varphi=0 \\
C \Delta \xi^{3 s-3}, & \text { for } \varphi=\varphi_{c} \\
C \Delta \xi^{3 s-2}, & \text { otherwise }\end{cases} \\
& C=\max _{\xi \neq \xi_{d}}\left(\left\|f_{0}^{2}\right\|,\left\|f_{0}^{\prime 2}\right\|, \ldots,\left\|\left(f_{0}^{(s-1)}\right)^{2}\right\|\right)
\end{aligned}
$$

where $f_{0}=f\left[u_{0}(\xi)\right]$ is the initial flux, $u_{0}(\xi)$ is the initial condition, $f_{0}^{(s-1)}$ is the $(s-1)$ th-order derivative of $f_{0}$ with respect to $\xi, s-1$ is the degree of the reconstruction polynomials, $\xi_{d}$ is a set of points at 


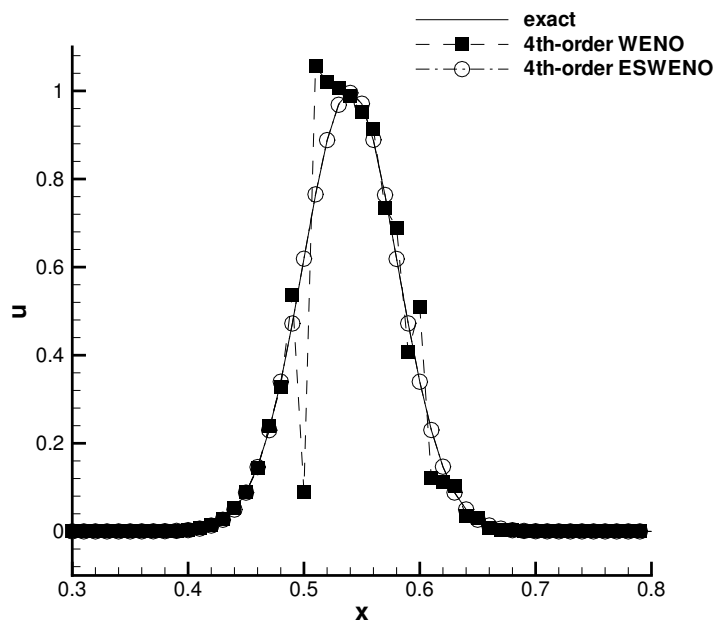

Figure 2: Solutions obtained with fourth-order WENO and ESWENO schemes on 101-point grid for linear wave equation with initial condition [eq. (81)] at $t=0.04$.

which the solution is discontinuous, and $\|\cdot\|$ is a norm in which the solution is sought. The scaling factor $C$ in equation (79) can easily be evaluated because it depends only on the initial condition for which the locations of all discontinuities are known a priori. Note that the parameter $\epsilon$ is calculated once and the same value is used over the entire time interval of integration; thus the computational cost does not increase.

In accordance with equation (23), the parameter $\delta_{i}$ should be scaled consistently with $\lambda_{i}$. Because $\lambda_{i}$ is a linear combination of the weights with each being of the order $O(1)$, the scaling factor of $\delta_{i}$ is set equal to one, which leads to

$$
\delta_{i}=\Delta \xi^{p-2 i+1} .
$$

Equations (79) and (80) eliminate the ambiguity in determining the parameters $\epsilon$ and $\delta_{i}$ for the ESWENO schemes; thus, the equations for $\epsilon$ and $\delta_{i}$ are free of tuning parameters. Furthermore, equations (79) and (80) are fully consistent with the sufficient conditions [eqs. (42) and (78)] and allow the ESWENO schemes to be invariant when the spatial and time variables are scaled by the same factor. Note that the parameter $\epsilon$ for the conventional WENO schemes of Jiang and Shu is set to $10^{-6}$ as recommended in [2]. In accordance with the recommendations of Borges et al. [8], the parameter $\epsilon$ for the fifth-order WENO scheme with the weights given by equations (17)-(19), and (22), which is referred as WENO-Z, is set to $10^{-40}$.

The time derivative for all steady test problems is approximated by using a third-order total variation diminishing (TVD) Runge-Kutta method that is developed in reference [11], while unsteady problems are integrated by using a fourth-order low-storage Runge-Kutta method (ref. [12]). To reduce the fourthorder temporal error component and make it consistent with the spatial error of fifth- and sixth-order schemes, the time step in global grid refinement studies is reduced by a factor of $2^{6 / 4}$ for each doubling of the number of grid points in space. The Courant-Friedrich-Levy (CFL) number has been set to 0.3 and 0.6 for the steady and unsteady test problems, respectively.

\subsection{Scalar Linear Wave Equation}

We begin by verifying that the new class of ESWENO schemes provide the design order of convergence for smooth problems, including local extrema. To check this property, we consider equation (1) with 
$a=1$ and the following initial condition:

$$
u_{0}(x)=e^{-300\left(x-x_{c}\right)^{2}},
$$

where $x_{c}$ is 0.5 . The computational domain for this test problem is set to $0 \leq x \leq 1$. Numerical solutions are calculated on a sequence of globally refined uniform grids and advanced in time up to $t=1$, which corresponds to one period in time.

First, we show that the conventional fourth-order WENO scheme is unstable for this smooth problem; however, the corresponding ESWENO is stable, and its solution is in excellent agreement with the exact solution, as shown in Figure 2. To make the conventional fourth-order WENO scheme stable, a

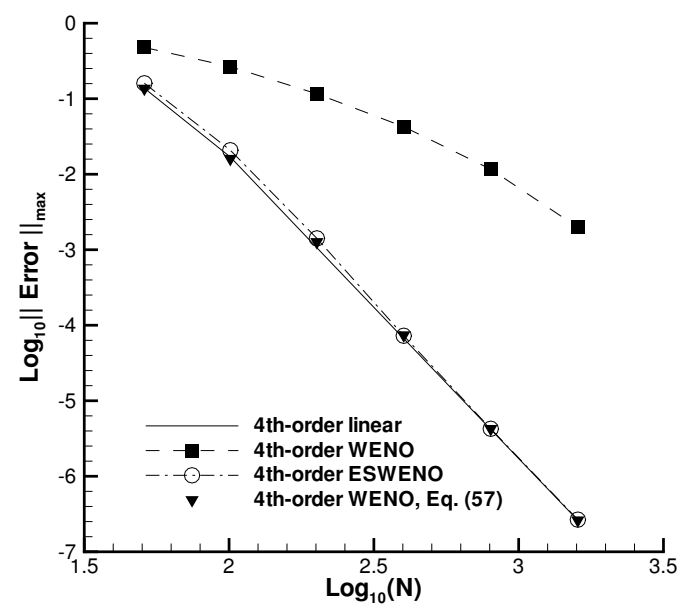

Figure 3: $L_{\infty}$ error norms obtained with the fourth-order linear, WENO, and ESWENO schemes for linear wave equation with initial condition given in equation (81).

smoothness indicator that corresponds to the downwind stencil has been modified as follows:

$$
\bar{\beta}^{R}=\left[\frac{\left(\beta^{L}\right)^{k}+\left(\beta^{C}\right)^{k}+\left(\beta^{R}\right)^{k}}{3}\right]^{1 / k},
$$

where $k$ is a constant that is greater than 1 . Note that $\bar{\beta}^{R}$ is a $C^{\infty}$ function of its arguments and approaches $\max \left(\beta^{L}, \beta^{C}, \beta^{R}\right)$ as $k \rightarrow \infty$. In contrast to equation (82), a modified smoothness indicator $\bar{\beta}^{R}=\max \left(\beta^{L}, \beta^{C}, \beta^{R}\right)$ that is proposed in reference [6] is a nonsmooth function of $\beta^{L}, \beta^{C}$ and $\beta^{R}$, which may lead to the degeneration of the design order of accuracy and is more prone to spurious oscillations near unresolved features and strong discontinuities. For $k \rightarrow \infty$, the downwind smoothness indicator that is given by equation (82) prevents the corresponding weight $w^{R}$ from being larger than the lesser of the other two weight functions; thus, the stencil is biased in the upwind direction near unresolved features. In smooth regions, all three smoothness indicators are of the same order, and the weights approach their preferred values of $w^{L}=\frac{1}{6}, w^{C}=\frac{2}{3}$, and $w^{R}=\frac{1}{6}$, which provides the design order of accuracy. For all of the numerical experiments that are presented herein, the parameter $k$ in equation (82) is equal to 4.

Figure 3 shows the $L_{\infty}$ error norms that are obtained with the fourth-order WENO and ESWENO schemes, and the corresponding underlying linear schemes. As shown in Figure 3, the fourth-order ESWENO scheme is significantly more accurate than the conventional fourth-order WENO scheme. The $L_{\infty}$ error that is obtained with the fourth-order ESWENO scheme is slightly higher than that obtained with the corresponding linear scheme on coarse meshes and reaches its theoretical limit starting at 

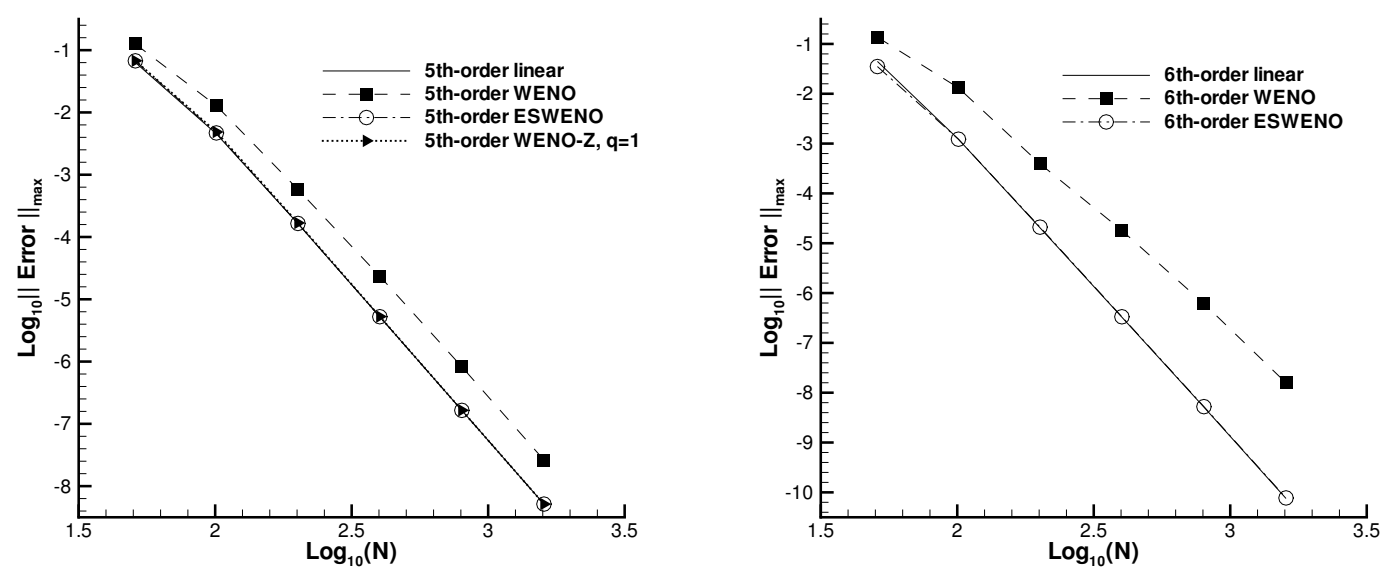

Figure 4: $L_{\infty}$ error norms obtained with the fifth- (left) and sixth-order (right) linear, WENO, WENO-Z, and ESWENO schemes for linear wave equation with initial condition given in equation (82).

$J=201$ grid points. The maximum error occurs at the peak of the Gaussian pulse indicating that the ESWENO scheme is design-order accurate at the smooth extrema. In contrast to the ESWENO scheme, the conventional WENO scheme demonstrates only a third-order convergence rate, even on the finest mesh with $J=1601$, and is two to three orders of magnitude less accurate on moderate and fine meshes.

If the new weights [eqs. (58) and (59)] are used instead of their conventional counterparts, then the design order of convergence of the fourth-order central WENO scheme is recovered, as shown in figure 3. Moreover, the fourth-order WENO scheme with the new weights provides slightly better accuracy on coarse meshes than the corresponding ESWENO scheme. This result is not surprising because the ESWENO scheme has the additional dissipation term [eq. (24)], which guarantees the stability. In general, if a WENO scheme with the new weights is stable, then it is slightly less dissipative than the corresponding ESWENO counterpart. For all of the problems that are considered, however, the results obtained with the conventional WENO schemes with the weights given by equations (58) and (59) are practically indistinguishable from those of the corresponding ESWENO schemes; therefore these results are not presented hereafter.

The $L_{\infty}$ error norms that are obtained with the fifth-order WENO-Z scheme and the fifth- and sixth-order WENO and ESWENO schemes for the same test problem are depicted in figure 4. Similar to the fourth-order case, the conventional sixth-order central WENO scheme is unstable. This problem is avoided by using the same upwinding technique that is outlined earlier. For the sixth-order central WENO scheme, the modified smoothness indicator that corresponds to the most downwind stencil is given by

$$
\bar{\beta}^{R R}=\left[\frac{\left(\beta^{L L}\right)^{k}+\left(\beta^{L}\right)^{k}+\left(\beta^{R}\right)^{k}+\left(\beta^{R R}\right)^{k}}{4}\right]^{1 / k} .
$$

Both the fifth-order WENO-Z and fifth- and sixth-order ESWENO schemes are equal in accuracy to the corresponding underlying linear schemes, as shown in Figure 4. Although the fifth-order WENO scheme exhibits the design-order convergence rate, its $L_{\infty}$ error norm is nearly an order of magnitude larger than those of the corresponding fifth-order WENO-Z and ESWENO schemes. In contrast to that of the sixth-order ESWENO scheme, the $L_{\infty}$ error that is obtained with the conventional sixth-order WENO scheme shows only fifth-order convergence and is quite far from the theoretical limit (represented by the corresponding sixth-order central linear scheme). Note that the WENO-Z schemes of fourth- and sixth-order are currently unavailable in the published literature and therefore are not presented herein.

As we have proven in sections 2 and 4.1, for the linear convection equation (1) with piecewise 


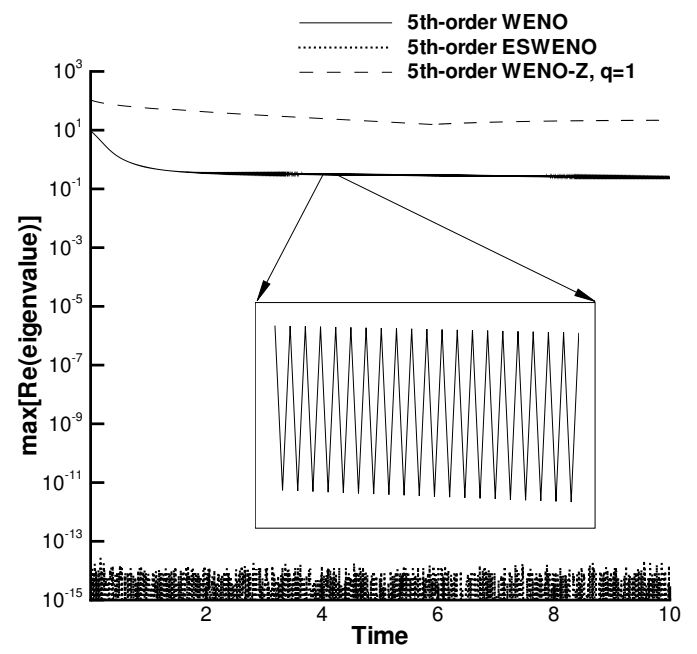

Figure 5: Time histories of rightmost eigenvalue of symmetric part of fifth-order WENO and ESWENO operators for Gaussian pulse problem.

continuous initial conditions, the family of ESWENO schemes is stable in the energy norm. The sufficient condition for stability is the negative semidefiniteness of $-\frac{1}{2}\left(\bar{D}+\bar{D}^{T}\right)$, where $\bar{D}$ is the ESWENO discrete operator. This property implies that all eigenvalues of the symmetric portion of the ESWENO operator are nonpositive. In contradistinction to the ESWENO scheme, the symmetric portion of the WENO operator may have positive eigenvalues. (See section 4.1.) Note that the same conclusion can be drawn for the fifth-order WENO-Z scheme (ref. [8]), whose derivative operator is identical to that of the conventional WENO scheme with the modified weights [eqs. (17)-(19), and (22)] which vary in the interval from 0 to 1 near the unresolved features. These properties of the WENO, WENO-Z, and ESWENO schemes are shown in figure 5, which gives the time histories of the rightmost eigenvalue of the symmetric portion of the operators computed on a 201-point grid. The rightmost eigenvalue of the ESWENO operator $-\frac{1}{2}\left(\bar{D}+\bar{D}^{T}\right)$ is equal to zero up to the order of the round-off error, while the symmetric part of the conventional fifth-order WENO and WENO-Z operators have positive eigenvalues of order $O\left(10^{-1}\right)$ and $O(10)$, respectively. (See figure 5.) This results from the fact that the symmetric portion of these WENO-type operators is not negative semidefinite, if unresolved features exist in the computational domain. Note that the presence of the positive eigenvalues does not imply that the fifth-order WENO and WENO-Z schemes are globally unstable because these eigenvalues correspond to different grid points at different moments of time.

The superiority of the ESWENO schemes with the new weights compared with the conventional and modified WENO counterparts, becomes evident when a more challenging problem is considered. In the previous test problem, the initial condition [eq. (81)] has the critical point at which $f^{\prime}=0$ and $f^{\prime \prime} \neq 0$ (i.e., the number of vanishing derivatives is $n_{v d}=1$ ). As shown in section 5.3, the fifth-order WENO-Z scheme that was developed by Borges et al. [8] fails to recover the design order of convergence if $n_{v d} \geq 6$ for an arbitrary choice of the parameter $m$ in equation (74). We now demonstrate this property for $n_{v d} \geq 3$. Consider equation (1) with the following initial condition:

$$
u_{0}(x)= \begin{cases}z^{18}-14 z^{16}+69 z^{14}-175 z^{12}+259 z^{10} & \text { for }|z| \leq 1 \\ -231 z^{8}+119 z^{6}-29 z^{4}+1, & \text { otherwise }\end{cases}
$$

where $z=5(x-0.5)$ and $0 \leq x \leq 1$. The above function is six times continuously differentiable and has three critical points: one at $x=0.5$ with $n_{v d}=3$ (i.e., $f^{\prime}(0.5)=f^{\prime \prime}(0.5)=f^{\prime \prime \prime}(0.5)=0$ and $\left.f^{\prime \prime \prime \prime}(0.5) \neq 0\right)$ and the remaining two at $x=0.3$ and 0.7 with $n_{v d}=6$. If we compare the $L_{\infty}$ error norms 


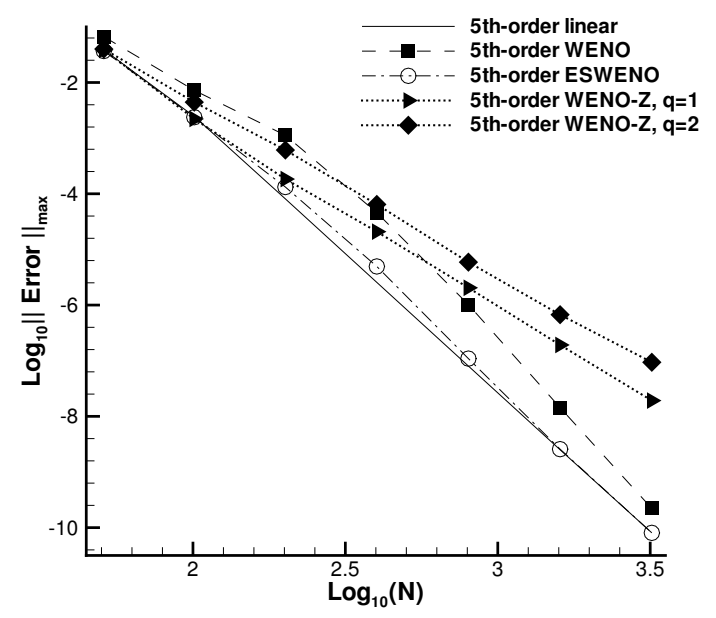

Figure 6: $L_{\infty}$ error norms obtained with fifth-order linear, WENO, WENO-Z, and ESWENO schemes for linear wave equation with initial conditions given in equation (84) at $t=1$.

computed with the fifth-order linear, WENO, WENO-Z, and ESWENO schemes at $t=1$, (see fig. 6), we see that the situation changes dramatically as compared with the previous test problem. As expected, the WENO-Z scheme fails to deliver fifth order convergence, while the ESWENO scheme is fifth-order accurate and provides practically the same error convergence as the underlying linear scheme. This result is not surprising because the parameter $\epsilon$ for the WENO-Z scheme is set to $10^{-40}$, as suggested in reference [8]. As a result, at a point $x=0.5+\Delta x$ the weights become of order $\mathrm{O}(1)$. Thus, if we use equations [(65), (22), and (74)] with $\epsilon=0$ and take into account that for the polynomial in equation (84), $f^{\prime}=O\left(\Delta x^{3}\right), f^{\prime \prime}=O\left(\Delta x^{2}\right), f^{\prime \prime \prime}=O(\Delta x)$, and $f^{\prime \prime \prime \prime}=O(1)$ at $x=0.5+\Delta x$, then we have

$$
w^{(r)}-d^{(r)}=O\left(\left[\frac{\left|\frac{13}{3} f^{\prime \prime} f^{\prime \prime \prime}-f^{\prime} f^{\prime \prime \prime \prime}\right| \Delta x^{5}}{f^{\prime 2} \Delta x^{2}+\left(\frac{13}{3} f^{\prime 2}-f^{\prime} f^{\prime \prime \prime}\right) \Delta x^{4}}\right]^{m}\right)=\left(\frac{O\left(\Delta x^{8}\right)}{O\left(\Delta x^{8}\right)}\right)^{m}=O(1) .
$$

As follows from the above estimate, the WENO-Z scheme fails to recover fifth order convergence regardless of the choice of the parameter $m$ in equation (74). Our numerical results corroborate this conclusion. Figure 6 shows that increasing the exponent $m$ in equation (74) results in an even larger deterioration in accuracy. In contrast to the WENO-Z scheme, the conventional fifth-order scheme of Jiang and Shu[2] converges at the design order and begins to approach the theoretical limit on the finest meshes. The main reason for such a behavior is the choice of the parameter $\epsilon$, which is set to $10^{-6}$. As the grid is refined, $\beta^{(r)} \rightarrow 0$, the denominator in equation (21) is dominated by $\epsilon$, and the weights approach their preferred values.

\subsection{The 1-D Euler Equations}

In reference [1], the third-order ESWENO scheme is proved to be energy-stable for a system of linear hyperbolic equations with periodic boundary conditions. This result can be directly extended to the class of higher order ESWENO schemes that are presented in this paper. For nonlinear conservation laws with nonperiodic boundary conditions, a similar proof for the high-order ESWENO schemes is not currently available. Nevertheless, we would like to test how the new schemes perform for the system of 
the quasi-1-D Euler equations, which are given by

$$
\begin{gathered}
\mathbf{U}=\left[\begin{array}{c}
\rho \\
\rho u \\
E
\end{array}\right], \quad \mathbf{F}=\left[\begin{array}{c}
\frac{\partial \mathbf{U}}{\partial t}+\frac{\partial \mathbf{F}}{\partial x}=\mathbf{G} \\
\rho u \\
\rho u^{2}+P \\
(E+P) u
\end{array}\right], \quad \mathbf{G}=-\frac{A_{x}}{A}\left[\begin{array}{c}
\rho u \\
\rho u^{2} \\
(E+P) u
\end{array}\right] \\
P=(\gamma-1)\left(E+\frac{\rho u^{2}}{2}\right),
\end{gathered}
$$

where $A=A(x)$ is the cross-sectional area of a quasi-1-D nozzle and $\gamma=1.4$. As has been shown in reference [1], the ESWENO reconstruction must be implemented in local characteristic fields to guarantee the stability of the ESWENO scheme for systems of hyperbolic conservation laws. In the present analysis, both the WENO and ESWENO reconstructions are based on the Lax-Friedrichs flux splitting. See reference [1] for further details.
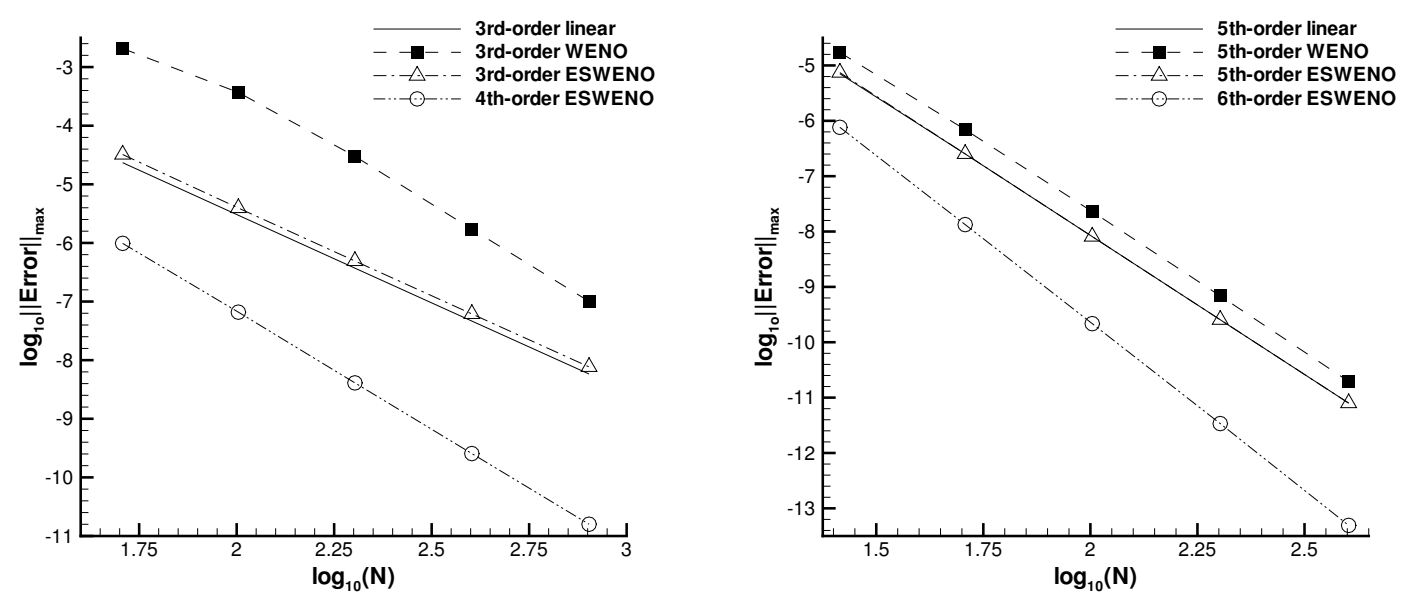

Figure 7: $L_{\infty}$ error norms obtained with the third- and fourth-order and fifth- and sixth-order linear, WENO, and ESWENO schemes for the subsonic quasi-1-D nozzle problem.

For the 1-D Euler equations, the preferred biasing of the stencil in the upwind direction, given by equations (82) and (83), is used for the central fourth- and sixth-order WENO and ESWENO schemes to suppress spurious oscillations near strong discontinuities. In the ESWENO case, these oscillations can also be eliminated by increasing the coefficient in front of the $D_{1} \Lambda_{1} D_{1}^{T}$ term in the artificial dissipation operator. However, this approach is more dissipative and also reduces the maximum CFL number for which the scheme remains stable. For the test problems that are presented in this section, the results obtained with the fifth-order WENO-Z scheme are practically indistinguishable from those of the fifthorder ESWENO scheme; therefore the WENO-Z results are not presented hereafter.

To verify that the new ESWENO schemes are design-order accurate for hyperbolic systems, we consider the steady-state isentropic flow through a quasi-1-D nozzle with the following cross-sectional area: $A(x)=1-0.8 x(1-x), 0 \leq x \leq 1$, as a test problem. The inflow Mach number is set to 0.5 and the pressure at $x=1$ is assumed to be equal to that at $x=0$. Under these conditions, the flow is fully subsonic, and the solution is smooth. Global grid-refinement studies for the third-, fourth-, fifth-, and sixth-order WENO and ESWENO schemes are presented in figure 7.

The $L_{\infty}$ error norms that are obtained with the third- and fourth-order ESWENO schemes exhibit the design order of convergence. On the finest mesh with $J=801$, the fourth-order ESWENO solution is approximately three and four orders of magnitude more accurate than those of the third-order ESWENO and WENO schemes, respectively. As shown in figure 7, the fifth-order ESWENO scheme provides the 
same accuracy as its underlying linear scheme, thereby exhibiting the perfect error convergence for this test problem. Although the conventional fifth-order WENO scheme exhibits the design order of convergence, its $L_{\infty}$ error norm is larger by a factor of 3 than that obtained with ESWENO counterpart. Similar to the fourth-order case, the central sixth-order ESWENO scheme converges at the design-order rate on both coarse and fine meshes, thus providing significantly more accurate solutions than both fifth-order schemes.

Remark 9. For this problem, the conventional central fourth- and sixth-order WENO schemes do not converge to a steady-state solution even with the upwinding mechanisms [eqs. (82) and (83)] turned on. The primary reason for this tendency is the presence of positive eigenvalues in the spectrum of the symmetric part of the conventional WENO operator.

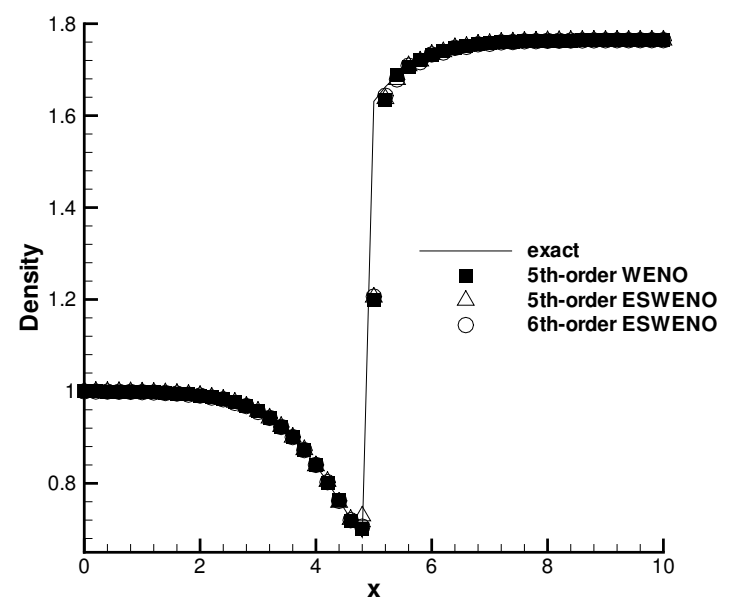

Figure 8: Comparison of fifth-order WENO and ESWENO schemes for steady transonic flow through quasi-1-D nozzle.

The next test problem is the steady transonic flow through a quasi-1-D nozzle with the following cross-sectional area:

$$
A(x)=1.398+0.347 \tanh (0.8 x-4), 0 \leq x \leq 10 .
$$

The Mach number at $x=0$ is 1.5 , and the outflow conditions have been chosen so that the shock is located at $x=5$. Density profiles are calculated using the fifth-order WENO and fifth- and sixth-order ESWENO schemes on a 51-point grid and are compared with the exact solution in figure 8. For all schemes, the captured shock is smeared over two grid cells, and the numerical solutions are essentially nonoscillatory and agree quite well with the exact solution. Note that the fifth- and sixth-order ESWENO schemes converge to the steady-state solution an order of magnitude faster than the conventional fifthorder WENO scheme; this result again indicates the presence of unstable modes that are generated by the positive eigenvalues of the WENO dissipation operator.

The last two problems considered are standard unsteady problems for testing shock-capturing schemes. The first problem is the Riemann problem with the initial conditions proposed by Sod[13]:

$$
(\rho, u, P)=\left\{\begin{array}{ll}
(1,0,1) & \text { if }-0.5 \leq x<0 \\
(0.125,0,0.1) & \text { if } 0 \leq x \leq 0.5
\end{array} .\right.
$$

The numerical solutions that are computed with both fourth- and sixth-order WENO and ESWENO schemes are essentially nonoscillatory, as one can see in figure 9. Note, however, that the fourth- and sixth-order ESWENO schemes provide better resolution near the contact discontinuity and the shock as compared with the conventional WENO schemes. 

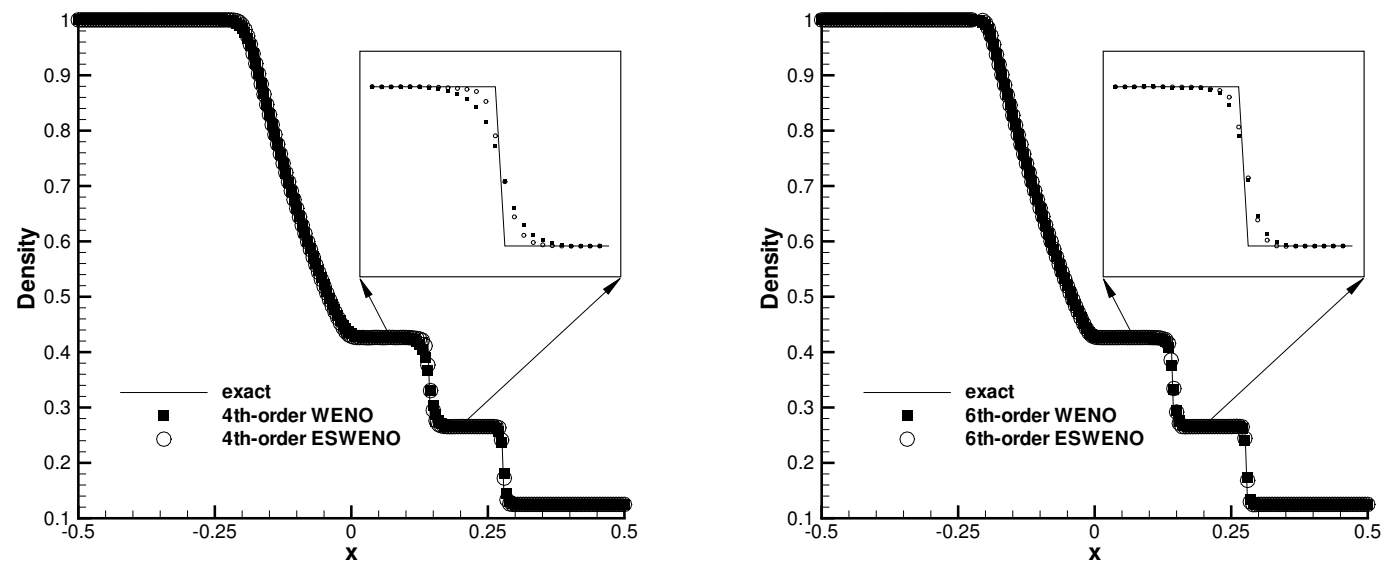

Figure 9: Density profiles computed with the fourth-order (left) and sixth-order (right) WENO and ESWENO schemes on a uniform grid with 201 points for the Sod problem at $t=0.16$.

We conclude this section by presenting the numerical results that are obtained with WENO and ESWENO schemes for the shock entropy-wave interaction problem. The solution of this benchmark problem contains both strong discontinuities and smooth structures, and is well suited for testing highorder shock-capturing schemes. The governing equations are the time-dependent 1-D Euler equations [eq. (86)] with $\mathbf{G}=\mathbf{0}$, subject to the following initial conditions:

$$
(\rho, u, p)=\left\{\begin{array}{lll}
(3.857134,2.629369,10.33333), & \text { if } \quad-5 \leq x<-4 \\
(1+0.2 \sin 5 x, 0,1), & \text { if } \quad-4 \leq x \leq 5 .
\end{array}\right.
$$

The governing equations are integrated in time up to $t=1.8$. The exact solution to this problem is not available. Therefore, a numerical solution that is obtained with the conventional fifth-order WENO scheme on a uniform grid with $J=4001$ grid points is used as a reference solution.

Numerical solutions that are computed with the fourth-, fifth-, and sixth-order WENO and ESWENO schemes on a 301-point grid at $t=1.8$ are compared with the "exact" reference solution in figure 10 . Both the WENO and ESWENO solutions are free of spurious oscillations. The fourth-order WENO scheme is the most dissipative among the considered schemes and has the worst resolution in a region just upstream of the moving shock. The fourth-order ESWENO scheme provides better resolution of the high-frequency oscillations behind the shock. However, the wave amplitudes are much lower than those that are computed with the fifth- and sixth-order schemes. Again, the fifth- and sixth-order ESWENO solutions are more accurate than those of the corresponding WENO schemes. Both ESWENO schemes resolve the smooth extrema quite well, and the solutions are essentially nonoscillatory near the captured shock.

\section{Conclusions}

A systematic methodology is developed to construct Energy Stable weighted essentially nonoscillatory (ESWENO) finite-difference schemes of arbitrary order, and is used to construct ESWENO schemes based on the WENO schemes that are presented in references [2] and [6]. The new ESWENO schemes differ from the conventional WENO schemes by the addition of a nonlinear artificial dissipation term of special form. The additional term is design-order accurate for smooth solutions, including smooth extrema, and guarantees that the ESWENO scheme is stable in the $L_{2}$-energy norm for both continuous and discontinuous solutions of hyperbolic systems; for the conventional WENO scheme, an energy 

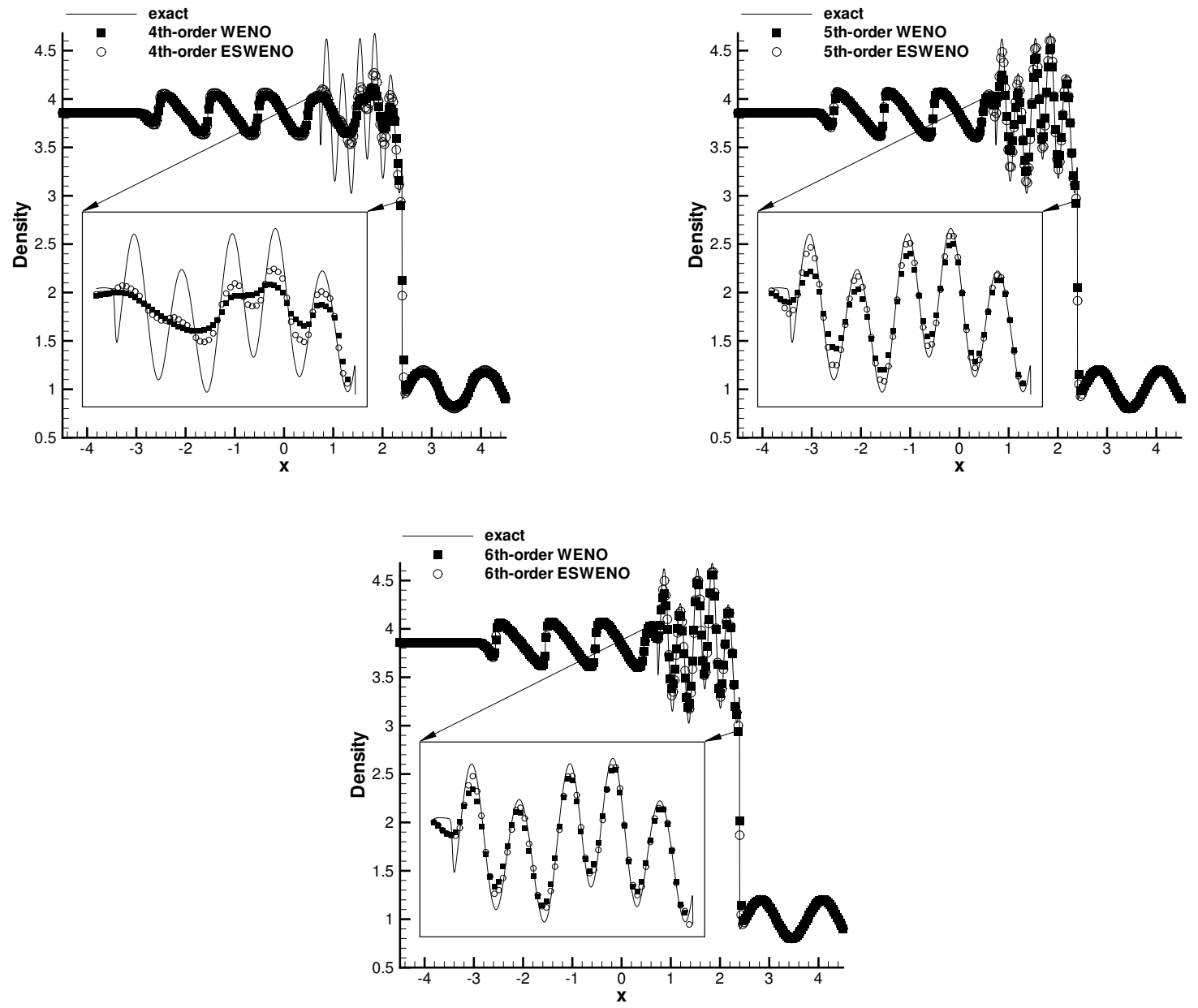

Figure 10: Density profiles computed with the fourth-order (top left), fifth-order (top right), and sixthorder (bottom) WENO and ESWENO schemes on uniform grid with 301 grid points for density sine wave/shock-interaction problem.

estimate is not available. The distinctive feature of the new class of ESWENO schemes is that the spectrum of the symmetric part of the ESWENO operator is always located in the left half-plane, while the symmetric part of the WENO operator has positive eigenvalues; thus, the conventional WENO schemes may become locally unstable.

New weight functions are also developed, guided by newly derived sufficient conditions guaranteeing design order accuracy for arbitrary WENO schemes; sufficient conditions that are valid regardless of the number of vanishing solution derivatives. A truncation error analysis is used to optimize the weight functions, so that the new ESWENO schemes satisfy the sufficient conditions for accuracy and provide essentially nonoscillatory solutions for problems with strong discontinuities. Numerical experiments confirm that the high-order ESWENO schemes with the new weights are significantly more accurate than conventional WENO schemes of the same order, and demonstrate excellent shock-capturing capabilities. 


\section{Appendix}

\subsection{Existence of Symmetric Decomposition}

The following lemma establishes the existence of the decomposition $D_{s y m}=\sum_{i=0}^{s}\left[D_{1}{ }^{i}\right] \Lambda_{i}\left[D_{1}{ }^{i}\right]^{T}$. The lemma provides an algorithm to decompose a matrix of arbitrary bandwidth $2 s+1$ into the sum of two matrices: one that can be expressed as one term of the desired symmetric form, and one of bandwidth $2(s-1)+1$.

Lemma 1. Define $E_{s}$ to be an $N$-dimensional, symmetric, bidiagonal matrix with the subdiagonal elements $e_{s+1,1}, e_{s+2,2}, \cdots, e_{N-1, N-s-1}, e_{N, N-s}$. The parameter $s$ is the offset from the main diagonal. Further, define $B_{s-1}$ to be an $N$-dimensional, symmetric, banded matrix of bandwidth $2(s-1)+1$ with elements that are functions of the matrix $E_{s}$. The matrices $E_{s}$ and $B_{s-1}$ satisfy the following relationship:

$$
E_{s}=(-1)^{s}\left[D_{1}^{s}\right] \Lambda_{s}\left[D_{1}^{s}\right]^{T}+B_{s-1} .
$$

with $\Lambda_{s}=\operatorname{diag}\left[e_{s+1,1}, e_{s+2,2}, \cdots, e_{N-1, N-s-1}, e_{N, N-s}, \mathbf{0}_{\mathbf{s}}^{T}\right]$ and $\mathbf{0}_{\mathbf{s}}$ is a zero vector of dimension $s$.

Proof: A proof by induction is not included herein. One step of the algorithm can be verified immediately by inspection.

A simple example illustrates Lemma 1 . Define the $5 \times 5$ matrices $E_{2}, D_{15}$, and $B_{1}$ such that $E_{2}=(-1)^{2}\left[D_{15}^{2}\right] \Lambda_{2}\left[D_{15}^{2}\right]^{T}+B_{1} \quad$ and

$$
\begin{gathered}
E_{2}=\left(\begin{array}{lllll}
0 & 0 & a & 0 & 0 \\
0 & 0 & 0 & b & 0 \\
a & 0 & 0 & 0 & c \\
0 & b & 0 & 0 & 0 \\
0 & 0 & c & 0 & 0
\end{array}\right) \quad ; \quad D_{15}=\left(\begin{array}{rrrrrr}
1 & 0 & 0 & 0 & -1 \\
-1 & 1 & 0 & 0 & 0 \\
0 & -1 & 1 & 0 & 0 \\
0 & 0 & -1 & 1 & 0 \\
0 & 0 & 0 & -1 & 1
\end{array}\right) \\
\Lambda_{2}=\left(\begin{array}{lllll}
a & 0 & 0 & 0 & 0 \\
0 & b & 0 & 0 & 0 \\
0 & 0 & c & 0 & 0 \\
0 & 0 & 0 & 0 & 0 \\
0 & 0 & 0 & 0 & 0
\end{array}\right) \quad ; \quad B_{1}=\left(\begin{array}{ccccc}
a & -2 a & 0 & 0 & 0 \\
-2 a & 4 a+b & -2(a+b) & 0 & 0 \\
0 & -2(a+b) & a+4 b+c & -2(b+c) & 0 \\
0 & 0 & -2(b+c) & b+4 c & -2 c \\
0 & 0 & 0 & -2 c & c
\end{array}\right)
\end{gathered}
$$

Remark 10. Lemma 1 can be used recursively, beginning with the outermost $s$ diagonal and working inward, to establish that $D_{\text {sym }}=\sum_{i=0}^{s}\left[D_{1}{ }^{i}\right] \Lambda_{i}\left[D_{1}{ }^{i}\right]^{T}$. Each step generates a new symmetric matrix with a bandwidth that is equal to 2 less than the previous value until only a diagonal matrix remains. Thus, the algorithm terminates after $s+1$ steps with the desired decomposition.

Remark 11. This recursive algorithm works for nonperiodic matrices in multiple spatial dimensions.

\subsection{ESWENO Schemes of Third- and Fourth-Order}

Based on equation (13), $\hat{f}_{j+\frac{1}{2}}$ defined for a third- or fourth-order WENO scheme is

$$
\hat{f}_{j+\frac{1}{2}}=w_{j+1 / 2}^{L} f_{j+1 / 2}^{L}+w_{j+1 / 2}^{C} f_{j+1 / 2}^{C}+w_{j+1 / 2}^{R} f_{j+1 / 2}^{R}
$$

where $w^{L}, w^{C}$, and $w^{R}$ are weight functions that are assigned to each respective stencil. The secondorder linear fluxes: $f_{j+1 / 2}^{(r)}$, defined in equation (88) for $r=\{L, C, R\}$, are

$$
\left(\begin{array}{c}
f^{L}\left(u_{j+1 / 2}\right) \\
f^{C}\left(u_{j+1 / 2}\right) \\
f^{R}\left(u_{j+1 / 2}\right)
\end{array}\right)=\frac{1}{2}\left(\begin{array}{cccc}
-1 & 3 & 0 & 0 \\
0 & 1 & 1 & 0 \\
0 & 0 & 3 & -1
\end{array}\right)\left(\begin{array}{c}
f\left(u_{j-1}\right) \\
f\left(u_{j}\right) \\
f\left(u_{j+1}\right) \\
f\left(u_{j+2}\right)
\end{array}\right)
$$


The terms $w^{L}, w^{C}$, and $w^{R}$ are the nonlinear weight functions that are given by equations (58) and (59) with

$$
\tau_{3}= \begin{cases}\left(-f_{j-1}+3 f_{j}-3 f_{j+1}+f_{j+2}\right)^{2}, & \text { for } \varphi \neq 0 \\ \left(f_{j-1}-2 f_{j}+f_{j+1}\right)^{2}, & \text { for } \varphi=0\end{cases}
$$

(See ref. [1] for further details). These weight functions have preferred values that are derived from underlying linear schemes, as well as solution-dependent components. The preferred values are given by the formulae

$$
d_{:}^{L}=\frac{1}{3}-\varphi \quad ; \quad d_{:}^{C}=\frac{2}{3} \quad ; \quad d_{:}^{R}=\varphi,
$$

where $\varphi$ is a parameter. The convergence rate of equation (13), with the preferred weight values that are given in equation (88), is equal to 3 for all values of the parameter $\varphi$ except $\varphi_{c}=\frac{1}{6}$, for which the convergence rate is 4 .

Combining equation (89) with equation (88) and substituting the resulting WENO flux $\hat{f}_{j+\frac{1}{2}}$ into equation (13) produces a stencil for the $j$ th grid point of the form

$$
D_{j, k}^{3}=\frac{1}{2 \Delta x}\left(\begin{array}{c|c|c|c|c}
0 & -w_{j+1 / 2}^{L} & 3 w_{j+1 / 2}^{L} & 0 & 0 \\
0 & 0 & w_{j+1 / 2}^{C} & w_{j+1 / 2}^{C} & 0 \\
0 & 0 & 0 & 3 w_{j+1 / 2}^{R} & -w_{j+1 / 2}^{R} \\
w_{j-1 / 2}^{L} & -3 w_{j-1 / 2}^{L} & 0 & 0 & 0 \\
0 & -w_{j-1 / 2}^{C} & -w_{j-1 / 2}^{C} & 0 & 0 \\
0 & 0 & -3 w_{j-1 / 2}^{R} & w_{j-1 / 2}^{R} & 0
\end{array}\right)\left(\begin{array}{c}
f\left(u_{j-2}\right) \\
f\left(u_{j-1}\right) \\
f\left(u_{j}\right) \\
f\left(u_{j+1}\right) \\
f\left(u_{j+2}\right)
\end{array}\right)
$$

with $k$ on the interval $j-2$ le $k \leq j+2$.

Simplifying $D^{3}$ using the expression

$$
w_{:}^{C}=1-w_{:}^{L}-w_{:}^{R}
$$

yields expressions for a single row of the $D^{3}$ matrix of the following form

$$
D_{j, k}^{3}=\frac{1}{2 \Delta x}\left(\begin{array}{c}
\vdots \\
0 \\
w_{j-1 / 2}^{L} \\
-2 w_{j-1 / 2}^{L}-w_{j+1 / 2}^{L}+w_{j-1 / 2}^{R}-1 \\
w_{j-1 / 2}^{L}+2 w_{j+1 / 2}^{L}-2 w_{j-1 / 2}^{R}-w_{j+1 / 2}^{R} \\
-w_{j+1 / 2}^{L}+w_{j-1 / 2}^{R}+2 w_{j+1 / 2}^{R}+1 \\
-w_{j+1 / 2}^{R} \\
0 \\
\vdots
\end{array}\right.
$$

The derivative matrix $D^{3}$ is now decomposed into symmetric and skew-symmetric parts

$$
D^{3}=D_{\text {skew }}^{3}+D_{\text {sym }}^{3} .
$$

As with the fifth-order case, the skew-symmetric component and the norm of $D^{3}$ take the forms

$$
D_{\text {skew }}^{3}=P^{-1} Q_{3} \quad ; \quad Q_{3}+Q_{3}^{T}=0 \quad ; \quad P=\Delta x I
$$

while the matrix $D_{\text {sym }}^{3}$ is expressed as

$$
D_{\text {sym }}^{3}=P^{-1}\left(D_{1}^{2} \Lambda_{2}^{3}\left[D_{1}^{2}\right]^{T}+D_{1}^{1} \Lambda_{1}^{3}\left[D_{1}^{1}\right]^{T}+D_{1}^{0} \Lambda_{0}^{3}\left[D_{1}^{0}\right]^{T}\right)
$$


where $\Lambda_{j}^{3}$ is a diagonal matrix with the expressions for the $j$ th element defined by

$$
\begin{gathered}
\left(\lambda_{2}^{3}\right)_{j, j}=\frac{1}{4}\left[w_{j+3 / 2}^{L}-w_{j+1 / 2}^{R}\right] \\
\left(\lambda_{1}^{3}\right)_{j, j}=\frac{1}{4}\left[-w_{j+3 / 2}^{L}+w_{j+1 / 2}^{L}-w_{j+1 / 2}^{R}+w_{j-1 / 2}^{R}\right] \\
\left(\lambda_{0}^{3}\right)_{j, j}=\frac{1}{2}\left[\begin{array}{l}
-w_{j-1 / 2}^{L}-w_{j-1 / 2}^{C}-w_{j-1 / 2}^{R} \\
+w_{j+1 / 2}^{L}+w_{j+1 / 2}^{C}+w_{j+1 / 2}^{R}
\end{array}\right]=0 .
\end{gathered}
$$

If we define $\left(\bar{\lambda}_{i}^{3}\right)_{j, j}, i=\overline{1,2}$, to be smoothly positive, then

$$
\left(\bar{\lambda}_{i}^{3}\right)_{j, j}=\frac{1}{2}\left[\sqrt{\left(\lambda_{i}^{3}\right)_{j, j}^{2}+\delta_{i}^{2}}-\left(\lambda_{i}^{3}\right)_{j, j}\right]
$$

and the additional artificial dissipation operator is given by

$$
\bar{D}_{a d}^{3}=P^{-1} \sum_{i=1}^{2}\left[D_{1}{ }^{i}\right]\left(\bar{\Lambda}_{i}^{3}\right)\left[D_{1}{ }^{i}\right]^{T},
$$

The resulting energy-stable scheme is obtained by adding the additional dissipation term to the corresponding conventional WENO scheme as

$$
\bar{D}^{3}=D^{3}+\bar{D}_{a d}^{3} .
$$

\subsection{ESWENO Schemes of Seventh- and Eighth-order}

The $\hat{f}_{j+\frac{1}{2}}$, defined for the seventh- and eighth-order WENO schemes, is

$$
\begin{aligned}
\hat{f}_{j+\frac{1}{2}} & =w_{j+1 / 2}^{L L} f_{j+1 / 2}^{L L}+w_{j+1 / 2}^{L} f_{j+1 / 2}^{L}+w_{j+1 / 2}^{C} f_{j+1 / 2}^{C} \\
& +w_{j+1 / 2}^{R} f_{j+1 / 2}^{R}+w_{j+1 / 2}^{R R} f_{j+1 / 2}^{R R}
\end{aligned}
$$

where $w^{L L}, w^{L}, w^{C}, w^{R}$, and $w^{R R}$ are weight functions that are assigned to each respective stencil, and are given by equations (58) and (59) with

$$
\tau_{7}= \begin{cases}\left(f_{j-3}-7 f_{j-2}+21 f_{j-1}-35 f_{j}+35 f_{j+1}-21 f_{j+2}+7 f_{j+3}-f_{j+4}\right)^{2}, & \text { for } \varphi \neq 0 \\ \left(-f_{j-3}+6 f_{j-2}-15 f_{j-1}+20 f_{j}-15 f_{j+1}+6 f_{j+2}-f_{j+3}\right)^{2}, & \text { for } \varphi=0\end{cases}
$$

The fourth-order linear fluxes $f_{j+1 / 2}^{(r)}$ for $r=\{L L, L, C, R, R R\}$, which are used in equation (97) are defined as

$$
\left(\begin{array}{c}
f^{L L}\left(u_{j+1 / 2}\right) \\
f^{L}\left(u_{j+1 / 2}\right) \\
f^{C}\left(u_{j+1 / 2}\right) \\
f^{R}\left(u_{j+1 / 2}\right) \\
f^{R R}\left(u_{j+1 / 2}\right)
\end{array}\right)=\frac{1}{12}\left(\begin{array}{cccccccc}
-3 & 13 & -23 & 25 & 0 & 0 & 0 & 0 \\
0 & 1 & -5 & 13 & 3 & 0 & 0 & 0 \\
0 & 0 & -1 & 7 & 7 & -1 & 0 & 0 \\
0 & 0 & 0 & 3 & 13 & -5 & 1 & 0 \\
0 & 0 & 0 & 0 & 25 & -23 & 13 & -3
\end{array}\right)\left(\begin{array}{c}
f\left(u_{j-3}\right) \\
f\left(u_{j-2}\right) \\
f\left(u_{j-1}\right) \\
f\left(u_{j}\right) \\
f\left(u_{j+1}\right) \\
f\left(u_{j+2}\right) \\
f\left(u_{j+3}\right) \\
f\left(u_{j+4}\right)
\end{array}\right)
$$

The derivative matrix $D^{7}$ is now decomposed into symmetric and skew-symmetric parts as

$$
D^{7}=D_{\text {skew }}^{7}+D_{\text {sym }}^{7} .
$$


As with the fifth-order case, the skew-symmetric component of $D^{7}$ takes the form

$$
D_{\text {skew }}^{7}=P^{-1} Q_{7} ; \quad Q_{7}+Q_{7}^{T}=0 ; \quad P=\Delta x I .
$$

The scheme converges with seventh-order accuracy if the weights are equal to their preferred values

$$
d_{:}^{L L}=\frac{1}{35}-\varphi, d_{:}^{L}=\frac{12}{35}-8 \varphi, d_{:}^{C}=\frac{18}{35}, d_{:}^{R}=\frac{4}{35}+8 \varphi, d_{:}^{R R}=\varphi
$$

and with eighth-order accuracy for the specific value $\varphi_{c}=\frac{1}{70}$.

The $D_{\text {sym }}^{7}$ can be simplified into the form

$$
\begin{aligned}
D_{\text {sym }}^{7} & =P^{-1}\left(D_{1}{ }^{4} \Lambda_{4}^{7}\left[D_{1}{ }^{4}\right]^{T}+D_{1}{ }^{3} \Lambda_{3}^{7}\left[D_{1}{ }^{3}\right]^{T}+D_{1}{ }^{2} \Lambda_{2}^{7}\left[D_{1}{ }^{2}\right]^{T}\right. \\
& \left.+D_{1}{ }^{1} \Lambda_{1}^{7}\left[D_{1}{ }^{1}\right]^{T}+D_{1}{ }^{0} \Lambda_{0}^{7}\left[D_{1}{ }^{0}\right]^{T}\right)
\end{aligned}
$$

where $\Lambda_{j}^{7}$ is a diagonal matrix with expressions for the $j$ th element defined by

$$
\begin{aligned}
& \left(\lambda_{4}^{7}\right)_{j, j}=\frac{1}{8}\left[w_{j+7 / 2}^{L L}-w_{j+1 / 2}^{R R}\right] \\
& \left(\lambda_{3}^{7}\right)_{j, j}=\frac{1}{24}\left[\quad+w_{j+5 / 2}^{L L}-9 w_{j+7 / 2}^{L L}+w_{j+5 / 2}^{L}\right. \\
& \left.-w_{j+1 / 2}^{R}+9 w_{j-1 / 2}^{R R}-w_{j+1 / 2}^{R R}\right] \\
& \left(\lambda_{2}^{7}\right)_{j, j}=\frac{1}{24}\left[\quad+2 w_{j+3 / 2}^{L L}-11 w_{j+5 / 2}^{L L}+9 w_{j+7 / 2}^{L L}\right. \\
& +2 w_{j+3 / 2}^{L}-2 w_{j+5 / 2}^{L} \\
& -w_{j+1 / 2}^{C}+w_{j+3 / 2}^{C} \\
& +2 w_{j-1 / 2}^{R}-2 w_{j+1 / 2}^{R} \\
& \left.-9 w_{j-3 / 2}^{R R}+11 w_{j-1 / 2}^{R R}-2 w_{j+1 / 2}^{R R}\right] \\
& \left(\lambda_{1}^{7}\right)_{j, j}=\frac{1}{24}\left[\quad+6 w_{i+1 / 2}^{L L}-13 w_{i+3 / 2}^{L L}+10 w_{i+5 / 2}^{L L}-3 w_{i+7 / 2}^{L L}\right. \\
& +3 w_{i+1 / 2}^{L}-4 w_{i+3 / 2}^{L}+w_{i+5 / 2}^{L} \\
& +w_{i-1 / 2}^{C}-w_{i+3 / 2}^{C} \\
& -w_{i-3 / 2}^{R}+4 w_{i-1 / 2}^{R}-3 w_{i+1 / 2}^{R} \\
& \left.+3 w_{i-5 / 2}^{R R}-10 w_{i-3 / 2}^{R R}+13 w_{i-1 / 2}^{R R}-6 w_{i+1 / 2}^{R R}\right] \\
& \left(\lambda_{0}^{7}\right)_{j, j}=\frac{1}{2}\left[\quad-w_{i-1 / 2}^{L L}-w_{i-1 / 2}^{L}-w_{i-1 / 2}^{C}-w_{i-1 / 2}^{R}-w_{i-1 / 2}^{R R}\right. \\
& \left.+w_{i+1 / 2}^{L L}+w_{i+1 / 2}^{L}+w_{i+1 / 2}^{C}+w_{i+1 / 2}^{R}+w_{i+1 / 2}^{R R}\right]=0 .
\end{aligned}
$$

If we Define $\left(\bar{\lambda}_{i}^{7}\right)_{j, j}, i=\overline{1,4}$, to be smoothly positive as

$$
\left(\bar{\lambda}_{i}^{7}\right)_{j, j}=\frac{1}{2}\left[\sqrt{\left(\lambda_{i}^{7}\right)_{j, j}^{2}+\delta_{i}^{2}}-\left(\lambda_{i}^{7}\right)_{j, j}\right]
$$

the additional artificial dissipation operator is given by

$$
\bar{D}_{a d}^{7}=P^{-1} \sum_{i=1}^{4}\left[D_{1}{ }^{i}\right]\left(\bar{\Lambda}_{i}^{7}\right)\left[D_{1}{ }^{i}\right]^{T},
$$

and the resulting energy-stable scheme is obtained by adding the additional dissipation term to the corresponding conventional WENO scheme as

$$
\bar{D}^{7}=D^{7}+\bar{D}_{a d}^{7} .
$$




\section{References}

[1] Yamaleev, N. K. and Carpenter, M. H., "Third-order energy stable WENO scheme," AIAA Paper 2008-2876, 2008.

[2] Jiang, G. and Shu, C.-W., "Efficient implementation of weighted ENO schemes," J. Comput. Phys., Vol. 126, 1996, pp. 202-228.

[3] Jiang, G. and Yu, S.-H., "Discrete shocks for finite difference approximations to scalar conservation laws," SIAM J. Numer. Anal., Vol. 35, No. 2, 1998, p. 749.

[4] Qiu, J.-M. and Shu, C.-W., "Convergence of Godunov-type schemes for scalar conservation laws under large time steps," to appear in J. Comput. Phys.

[5] Wang, Z. J. and Chen, R. F., "Optimized weighted essentially nonoscillatory schemes for linear waves with discontinuity," J. Comput. Phys., Vol. 174, 2001, pp. 381-404.

[6] Martin, M. P., Taylor, E. M., Wu, M., and Weris, V. G., "A bandwidth-optimized WENO scheme for the effective direct numerical simulation of compressible turbulence," J. Comput. Phys., Vol. 220, 2006, pp. 270-289.

[7] Hendrick, A. K., Aslam, T. D., and Powers, J. M., "Mapped weighted essentially non-oscillatory schemes: Achieving optimal order near critical points," J. Comput. Phys., Vol. 207, 2005, pp. $542-567$.

[8] Borges, R., Carmona, M., Costa, B., and Don, W. S., "An improved weighted essentially nonoscillatory scheme for hyperbolic conservation laws," J. of Comput. Phys., Vol. 227, 2008, pp. 3191-3211.

[9] Carpenter, M. H., Nordström, J., and Gottlieb, D., "A stable and conservative interface treatment of arbitrary spatial accuracy," J. Comput. Phys., Vol. 148, 1999, pp. 341-365.

[10] Kreiss, H. O. and Scherer, G., "Finite element and finite difference methods for hyperbolic partial differential equations," Mathematical Aspects of Finite Elements in Partial Differential Equations, Academic Press, New York, 1974.

[11] Shu, C.-W., "Total-variation-diminishing time discretizations," SIAM J. Sci. Stat. Comput., Vol. 9, 1988, p. 1073.

[12] Kennedy, C. A., Carpenter, M. H., and Lewis, R., "Low-storage, explicit Runge-Kutta schemes for the compressible Navier-Stokes equations," Appl. Numer. Math., Vol. 35, No. 3, 2000, pp. 177-219.

[13] Sod, G. A., "A survey of several finite difference methods for systems of nonlinear hyperbolic conservation laws," J. Comput. Phys., Vol. 43, 1978, pp. 1-31. 

The public reporting burden for this collection of information is estimated to average 1 hour per response, including the time for reviewing instructions, searching existing data sources, gathering and maintaining the data needed, and completing and reviewing the collection of information. Send comments regarding this burden estimate or any other aspect of this collection of information, including suggestions for reducing this burden, to Department of Defense, Washington Headquarters Services, Directorate for Information Operations and Reports (0704-0188), 1215 Jefferson Davis Highway, Suite 1204, Arlington, VA 22202-4302. Respondents should be aware that notwithstanding any other provision of law, no person shall be subject to any penalty for failing to comply with a collection of information if it does not display a currently valid OMB control number.

PLEASE DO NOT RETURN YOUR FORM TO THE ABOVE ADDRESS.

\begin{tabular}{|l|l|l|l}
\hline 1. REPORT DATE $(D D-M M-Y Y Y Y)$ & 2. REPORT TYPE & 3. DATES COVERED (FrOm - TO)
\end{tabular}

\begin{tabular}{l|l} 
01-10-2008 & Technical Memorandum \\
\hline
\end{tabular}

\section{TITLE AND SUBTITLE}

A Systematic Methodology for Constructing High-Order Energy-Stable WENO

Schemes 5a. CONTRACT NUMBER

5b. GRANT NUMBER

5c. PROGRAM ELEMENT NUMBER

\section{AUTHOR(S)}

\section{5d. PROJECT NUMBER}

Nail K. Yamaleev and Mark H. Carpenter

\section{5e. TASK NUMBER}

\section{5f. WORK UNIT NUMBER}

599489.02.07.07.04.03.01

\section{PERFORMING ORGANIZATION NAME(S) AND ADDRESS(ES)}

NASA Langley Research Center

Hampton, VA 23681-2199

\section{SPONSORING/MONITORING AGENCY NAME(S) AND ADDRESS(ES)}

National Aeronautics and Space Administration

Washington, DC 20546-0001

\section{DISTRIBUTION/AVAILABILITY STATEMENT}

Unclassified-Unlimited

Subject Category 64

Availability: NASA CASI (301) 621-0390

\section{SUPPLEMENTARY NOTES}

An electronic version can be found at http://ntrs.nasa.gov.

\section{ABSTRACT}

A third-order Energy Stable Weighted Essentially Non-Oscillatory (ESWENO) finite difference scheme developed by Yamaleev and Carpenter (AIAA 2008-2876, 2008) was proven to be stable in the energy norm for both continuous and discontinuous solutions of systems of linear hyperbolic equations. Herein, a systematic approach is presented that enables "energy stable" modifications for existing WENO schemes of any order. The technique is demonstrated by developing a one-parameter family of fifth-order upwind-biased ESWENO schemes; ESWENO schemes up to eighth order are presented in the appendix. New weight functions are also developed that provide (1) formal consistency, (2) much faster convergence for smooth solutions with an arbitrary number of vanishing derivatives, and (3) improved resolution near strong discontinuities.

\section{SUBJECT TERMS}

high-order, finite-difference, weighted essentially non-oscillatory, numerical stability, artificial dissipation

\begin{tabular}{|l|c|c|}
\hline 16. SECURITY CLASSIFICATION OF: \\
\hline a. REPORT & b. ABSTRACT & c. THIS PAGE \\
$\mathrm{U}$ & $\mathrm{U}$ & $\mathrm{U}$ \\
\hline
\end{tabular}

17. LIMITATION OF
ABSTRACT
UU

\begin{tabular}{l|l|}
$\begin{array}{l}\text { 18. NUMBER } \\
\text { OF } \\
\text { PAGES }\end{array}$ & $\begin{array}{l}\text { 19a. NAME OF RESPONSIBLE PERSON } \\
\text { STI Help Desk (email: help@sti.nasa.gov) }\end{array}$ \\
\cline { 2 - 2 } 38 & $\begin{array}{l}\text { 19b. TELEPHONE NUMBER (Include area code) } \\
(301) 621-0390\end{array}$ \\
\hline
\end{tabular}





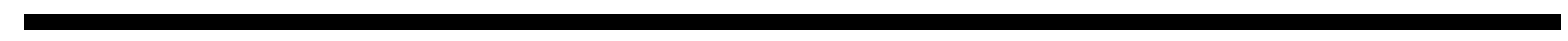

